arXiv version: fonts, pagination and layout may vary from GTM published version

\title{
Quasi-polynomials and the Bethe Ansatz
}

\author{
E MUKHIN \\ A VARCHENKO
}

\begin{abstract}
We study solutions of the Bethe Ansatz equation related to the trigonometric Gaudin model associated to a simple Lie algebra $\mathfrak{g}$ and a tensor product of irreducible finite-dimensional representations. Having one solution, we describe a construction of new solutions. The collection of all solutions obtained from a given one is called a population. We show that the Weyl group of $\mathfrak{g}$ acts on the points of a population freely and transitively (under certain conditions).

To a solution of the Bethe Ansatz equation, one assigns a common eigenvector (called the Bethe vector) of the trigonometric Gaudin operators. The dynamical Weyl group projectively acts on the common eigenvectors of the trigonometric Gaudin operators. We conjecture that this action preserves the set of Bethe vectors and coincides with the action induced by the action on points of populations. We prove the conjecture for $s l_{2}$.
\end{abstract}

82B23; 17B67

\section{Introduction}

The Bethe Ansatz is a method to diagonalize a commuting family of linear operators, usually called Hamiltonians. The method is applied to Hamiltonians of numerous quantum integrable systems. Given a solution of a suitable system of equations (called the Bethe Ansatz equation), the Bethe Ansatz produces an eigenvector (called the Bethe vector). This paper is motivated by the Bethe Ansatz method applied to the trigonometric Gaudin model, see Markov, Schechtman and the second author [5, 15], and Section 5.

For the case of the trigonometric Gaudin model the Bethe Ansatz equation and the Bethe vectors depend on an additional parameter, a generic $\mathfrak{g}$-weight $\lambda$. The Bethe Ansatz equation has the form (3). The Bethe vectors have the form (15), see Proposition 5.1. The Bethe Ansatz equation (3) can be formulated as a system of suitable Wronskian equations for a tuple of polynomials $y=\left(y_{1}, \ldots, y_{r}\right)$ of one variable, where $r$ is the rank of $\mathfrak{g}$ and the polynomials are labeled by simple roots of $\mathfrak{g}$, see Theorem 3.5. 
For example, let $\mathfrak{g}=s l_{2}$. The $s l_{2}$-weights can be identified with complex numbers. Consider the trigonometric Gaudin model associated to the tensor product of irreducible $s l_{2}$-modules with highest weights $\Lambda_{j}$, located respectively at points $z_{j}$. In this case, the Bethe Ansatz equation with parameter $\lambda \in \mathbb{C}$ is an equation on one polynomial $y$. The polynomial $y$ satisfies the Bethe Ansatz equation, if and only if its roots are simple and there exists another polynomial $\tilde{y}$ such that

$$
y^{\prime}\left(x^{\lambda+1} \tilde{y}\right)-y\left(x^{\lambda+1} \tilde{y}\right)^{\prime}=x^{\lambda} \prod_{j}\left(x-z_{j}\right)^{\Lambda_{j}} .
$$

For a given $y$ and a non-integer $\lambda$, the polynomial $\tilde{y}$ is unique. One can show that for almost all $\lambda$, the roots of $\tilde{y}$ are simple. Moreover, if the roots of $\tilde{y}$ are simple, then the polynomial $\tilde{y}$ also satisfy the Bethe Ansatz equation but with the new parameter $-\lambda-2$. Thus from one solution of the Bethe Ansatz equation with parameter $\lambda$ (the polynomial $y$ ) we obtain another solution with parameter $-\lambda-2$ (the polynomial $\tilde{y}$ ). We call this procedure the simple reproduction procedure.

For an arbitrary simple Lie algebra $\mathfrak{g}$, there is a similar simple reproduction procedure associated with every simple root of $\mathfrak{g}$. Consider an $r$-tuple $y=\left(y_{1}, \ldots, y_{r}\right)$ of polynomials forming a solution of the Bethe Ansatz equation associated with a generic $\mathfrak{g}$-weight $\lambda$. Then we have the $i$-th simple reproduction procedure for $i=1, \ldots, r$. The $i$-th simple reproduction procedure constructs a new tuple $y^{(i)}=\left(y_{1}, \ldots, y_{i-1}, \tilde{y}_{i}, y_{i+1}, \ldots, y_{r}\right)$ under certain conditions.

We call an $r$-tuple of polynomials $y=\left(y_{1}, \ldots, y_{r}\right)$ fertile with respect to $\lambda$ if the $i$-th simple reproduction procedure is well-defined for $i=1, \ldots, r$. In particular, if $y$ forms a solution of the Bethe Ansatz equation associated to $\lambda$, then $y$ is fertile with respect to $\lambda$. Moreover, if the $i$-th simple reproduction procedure results in a generic (in an appropriate sense) $r$-tuple $y^{(i)}$, then $y^{(i)}$ also forms a solution of the Bethe Ansatz equation associated to the weight $s_{i} \cdot \lambda$, where $s_{i}$ is the $i$-th elementary reflection in the Weyl group of $\mathfrak{g}$. It follows that the $r$-tuple $y^{(i)}$ is fertile with respect to $s_{i} \cdot \lambda$.

We call an $r$-tuple of polynomials $y$ super-fertile with respect to $\lambda$ if all iterations of the simple reproduction procedures are well defined. We conjecture that if $y$ forms a solution of the Bethe Ansatz equation then $y$ is super-fertile. We prove the conjecture for simple Lie algebras of type $A_{r}, B_{r}$.

The set of all $r$-tuples obtained from a given super-fertile $r$-tuple by iterations of simple reproduction procedures is called a population.

For simple Lie algebras, we prove that the population obtained from a super-fertile $r$-tuple associated to a generic weight $\lambda$ contains exactly one $r$-tuple associated to 
every weight of the form $w \cdot \lambda$, where $w$ runs through the elements of the Weyl group of $\mathfrak{g}$. We also prove that the population does not contain any other $r$-tuples. This one-to-one correspondence between the tuples of the population and the weights of the form $w \cdot \lambda$, allows us to introduce a free and transitive action of the Weyl group on points of the population. Then the action of simple reflections is given by the simple reproduction procedures. The proof is based on the important fact that in the case of $A_{r}$, the populations are in one-to-one correspondence with certain spaces of quasi-polynomials, see Corollary 4.4.

If all elements of a population are generic and therefore correspond to solutions of the Bethe Ansatz equation, then we have an action of the Weyl group on the set of the solutions. In particular this defines an action of the Weyl group on the set of the associated Bethe vectors, considered up to proportionality.

On the other hand, the dynamical Weyl group commutes with the trigonometric Gaudin operators and projectively acts on eigenvectors of the trigonometric Gaudin operators, see Tarasov and the second author [18] and Lemma 5.5.

We conjecture that the action of the dynamical Weyl group maps the Bethe vectors to (scalar multiples of) the Bethe vectors and moreover, the two actions on the Bethe vectors coincide. We prove this conjecture for $\mathfrak{g}=s l_{2}$.

The reproduction procedure exists for solutions of the Bethe Ansatz equations associated with many quantum integrable models. In Sections 6, 7 we give two other examples of the situation in which the corresponding Bethe Ansatz equation admits a reproduction procedure and prove that the elements of the corresponding populations are also labeled by the elements of the Weyl group. These examples are related to the quasi-periodic Gaudin and XXX models. In joint work with Tarasov [7], we apply the results of Sections 6 and 7 to study the Bethe Ansatz of $\left(g l_{N}, g l_{M}\right)$-dual quasiperiodic Gaudin and XXX models.

The notions of the reproduction procedure and populations for the Bethe Ansatz equation of the Gaudin and XXX-type models were introduced in our papers [9, 13], see also $[8,10]$. The populations in that situation are shown to be isomorphic to the flag variety of the Langlands dual algebra $\mathfrak{g}^{\vee}$ for all simple Lie algebras in the case of the Gaudin model by Frenkel [3] and the authors [11], and for the XXX-type model by the authors [10].

The paper is constructed as follows. Section 2 contains notation and definitions. In Section 3 we define the reproduction procedure and populations. In Section 4 we prove that for simple Lie algebras, the Weyl group acts freely and transitively on the elements 
of a population. In Section 5 we discuss two actions of the Weyl group on the Bethe vectors, the one given by the action of the dynamical Weyl groups and the one given by the reproduction procedure. We compare them in the case of $s l_{2}$. In Sections 6 and 7 we describe two more examples of the situation in which the Bethe Ansatz admit a reproduction procedure and the the Weyl group acts freely and transitively on the elements of a population.

Acknowledgements We thank V Tarasov for many valuable discussions.

Research of EM is supported in part by NSF grant DMS-0601005. Research of AV is supported in part by NSF grant DMS-0244579.

\section{Master functions and critical points}

\subsection{Kac-Moody algebras}

Let $A=\left(a_{i j}\right)_{i, j=1}^{r}$ be a generalized Cartan matrix, $a_{i i}=2, a_{i j}=0$ if and only $a_{j i}=0$, $a_{i j} \in \mathbb{Z}_{\leq 0}$ if $i \neq j$. We assume that $A$ is symmetrizable, there is a diagonal matrix $D=\operatorname{diag}\left\{d_{1}, \ldots, d_{r}\right\}$ with positive integers $d_{i}$ such that $B=D A$ is symmetric.

Let $\mathfrak{g}=\mathfrak{g}(A)$ be the corresponding complex Kac-Moody Lie algebra (see Kac [4, Section 1.2]), $\mathfrak{h} \subset \mathfrak{g}$ the Cartan subalgebra. The associated scalar product is nondegenerate on $\mathfrak{h}^{*}$ and $\operatorname{dim} \mathfrak{h}=r+2 d$, where $d$ is the dimension of the kernel of the Cartan matrix $A$.

Let $\alpha_{i} \in \mathfrak{h}^{*}, \alpha_{i}^{\vee} \in \mathfrak{h}, i=1, \ldots, r$, be the sets of simple roots, coroots, respectively. We have

$$
\begin{aligned}
\left(\alpha_{i}, \alpha_{j}\right) & =d_{i} a_{i j}, \\
\left\langle\lambda, \alpha_{i}^{\vee}\right\rangle & =2\left(\lambda, \alpha_{i}\right) /\left(\alpha_{i}, \alpha_{i}\right), \quad \lambda \in \mathfrak{h}^{*} .
\end{aligned}
$$

Let $\mathcal{P}=\left\{\lambda \in \mathfrak{h}^{*} \mid\left\langle\lambda, \alpha_{i}^{\vee}\right\rangle \in \mathbb{Z}\right\}$ and $\mathcal{P}^{+}=\left\{\lambda \in \mathfrak{h}^{*} \mid\left\langle\lambda, \alpha_{i}^{\vee}\right\rangle \in \mathbb{Z}_{\geq 0}\right\}$ be the sets of integral and dominant integral weights.

Fix $\rho \in \mathfrak{h}^{*}$ such that $\left\langle\rho, \alpha_{i}^{\vee}\right\rangle=1, i=1, \ldots, r$. We have $\left(\rho, \alpha_{i}\right)=\left(\alpha_{i}, \alpha_{i}\right) / 2$.

The Weyl group $\mathcal{W} \in \operatorname{End}\left(\mathfrak{h}^{*}\right)$ is generated by reflections $s_{i}, i=1, \ldots, r$,

$$
s_{i}(\lambda)=\lambda-\left\langle\lambda, \alpha_{i}^{\vee}\right\rangle \alpha_{i}, \quad \lambda \in \mathfrak{h}^{*} .
$$

We use the notation

$$
w \cdot \lambda=w(\lambda+\rho)-\rho, \quad w \in \mathcal{W}, \lambda \in \mathfrak{h}^{*},
$$

for the shifted action of the Weyl group. 


\subsection{The definition of master functions and critical points}

We fix a Kac-Moody algebra $\mathfrak{g}=\mathfrak{g}(A)$. We fix $\Lambda=\left(\Lambda_{i}\right)_{i=1}^{n}, \Lambda_{i} \in \mathcal{P}^{+} ; z=\left(z_{i}\right)_{i=1}^{n} \in$ $\mathbb{C}^{n}$. We assume $z_{i} \neq 0$ and $z_{i} \neq z_{j}$ if $i \neq j$. The parameters $\Lambda, z$ are always fixed and we often do not stress the dependence of our objects on these parameters.

In addition we choose $\lambda \in \mathfrak{h}^{*}$ and $l=\left(l_{1}, \ldots, l_{r}\right) \in \mathbb{Z}_{\geq 0}^{r}$. The choice of $l$ is equivalent to the choice of the weight at infinity $\Lambda_{\infty}$ defined by the formula:

$$
\Lambda_{\infty}=\sum_{s=1}^{n} \Lambda_{s}-\sum_{i=1}^{r} l_{i} \alpha_{i} \in \mathcal{P} .
$$

The master function $\Phi\left(t ; \Lambda_{\infty}, \lambda\right)$ is defined as follows (see Felder, Schechtman and the second author $[2,15])$ :

$$
\begin{gathered}
\Phi\left(t ; \Lambda_{\infty}, \lambda\right)=\prod_{i=1}^{r} \prod_{j=1}^{l_{i}}\left(t_{j}^{(i)}\right)^{-\left(\lambda, \alpha_{i}\right)} \prod_{i=1}^{r} \prod_{j=1}^{l_{i}} \prod_{s=1}^{n}\left(t_{j}^{(i)}-z_{s}\right)^{-\left(\Lambda_{s}, \alpha_{i}\right)} \times \\
\prod_{i=1}^{r} \prod_{1 \leq j<s \leq l_{i}}\left(t_{j}^{(i)}-t_{s}^{(i)}\right)^{\left(\alpha_{i}, \alpha_{i}\right)} \prod_{1 \leq i<j \leq r} \prod_{s=1}^{l_{i}} \prod_{k=1}^{l_{j}}\left(t_{s}^{(i)}-t_{k}^{(j)}\right)^{\left(\alpha_{i}, \alpha_{j}\right)} .
\end{gathered}
$$

The master function $\Phi$ is a function of variables $t=\left(t_{j}^{(i)}\right)_{i=1, \ldots, r}^{j=1, \ldots, l_{i}}$.

The master function $\Phi$ is symmetric with respect to permutations of variables with the same upper index.

A point $t$ with complex coordinates is called a critical point associated to $\left(\Lambda, z ; \Lambda_{\infty}, \lambda\right)$ (we often write just $\left(\Lambda_{\infty}, \lambda\right)$ ) if the following system of algebraic equations is satisfied

$$
-\frac{\left(\lambda, \alpha_{i}\right)}{t_{j}^{(i)}}-\sum_{s=1}^{n} \frac{\left(\Lambda_{s}, \alpha_{i}\right)}{t_{j}^{(i)}-z_{s}}+\sum_{s, s \neq i} \sum_{k=1}^{l_{s}} \frac{\left(\alpha_{s}, \alpha_{i}\right)}{t_{j}^{(i)}-t_{k}^{(s)}}+\sum_{s, s \neq j} \frac{\left(\alpha_{i}, \alpha_{i}\right)}{t_{j}^{(i)}-t_{s}^{(i)}}=0
$$

where $i=1, \ldots, r, j=1, \ldots, l_{i}$. In other words, a point $t$ is a critical point if

$$
\left(\Phi^{-1} \frac{\partial \Phi}{\partial t_{j}^{(i)}}\right)\left(t ; \Lambda_{\infty}, \lambda\right)=0, \quad i=1, \ldots, r, j=1, \ldots, l_{i} .
$$

Note that the product of symmetric groups $S_{l}=S_{l_{1}} \times \cdots \times S_{l_{r}}$ acts on the critical set of the master function permuting the coordinates with the same upper index. All orbits have the same cardinality $l_{1} ! \ldots l_{r} !$. We make no distinction between critical points in the same orbit.

The system of equations (3) coincides with the Bethe Ansatz equation of the nonhomogeneous Gaudin model, see Reshetikhin and the second author [14] and Proposition 5.1. 
Lemma 2.1 For almost all values of $\lambda$, the set of critical points associated to $\left(\Lambda_{\infty}, \lambda\right)$ is finite.

Proof The lemma follows from [9, Lemma 2.1].

\section{Populations}

\subsection{The $s l_{2}$ populations}

In the case of $s l_{2}$ the dominant integral weights are identified with non-negative integers and the system of equations (3) takes the form:

$$
-\frac{\lambda}{t_{j}}-\sum_{s=1}^{n} \frac{\Lambda_{s}}{t_{j}-z_{s}}+\sum_{k, k \neq j}^{l} \frac{2}{t_{j}-t_{k}}=0,
$$

$j=1, \ldots, l$, where $\Lambda_{s} \in \mathbb{Z}_{>0}$ and $\lambda \in \mathbb{C}$. The weight $\Lambda_{\infty}$ is given by $\Lambda_{\infty}=$ $\sum_{s=1}^{n} \Lambda_{s}-2 l$.

We set

$$
T(x)=\prod_{s=1}^{n}\left(x-z_{s}\right)^{\Lambda_{s}}, \quad F=x \prod_{s=1}^{n}\left(x-z_{s}\right) .
$$

Given a tuple $t=\left(t_{1}, \ldots, t_{l}\right)$ we represent it by the polynomial $y(x)=\prod_{j=1}^{l}\left(x-t_{j}\right)$. We are interested in the zeros of $y(x)$ and therefore we make no distinction between $y(x)$ and $c y(x)$, where $c$ is a non-zero constant.

A polynomial $y(x)$ is called off-diagonal with respect to $(\Lambda, z)$ if $y(x)$ has only simple roots, $y(0) \neq 0$ and $y\left(z_{s}\right) \neq 0$ for all $s=1, \ldots, n$ such that $\Lambda_{s} \neq 0$.

Since $(\Lambda, z)$ is fixed, we often call polynomials $y(x)$ off-diagonal with respect to $(\Lambda, z)$ simply off-diagonal.

Lemma 3.1 (T J Stieltjes [17, Section 6.81]) A polynomial $y$ of degree $l$ represents an $s l_{2}$ critical point associated to $\left(\Lambda_{\infty}, \lambda\right)$ if and only if $y$ is off-diagonal and there exists a polynomial $C(x)$ such that

$$
F(x) y^{\prime \prime}-F(x) \ln ^{\prime}\left(x^{\lambda} T(x)\right) y^{\prime}(x)+C(x) y(x)=0 .
$$

Proof Equation (4) can be reformulated as the statement that the function $y^{\prime \prime}-\ln ^{\prime}\left(x^{\lambda} T\right) y^{\prime}$ equals zero at $x=t_{j}$ for all $j$. Therefore (4) is equivalent to the divisibility of the polynomial $F y^{\prime \prime}-F \ln ^{\prime}\left(x^{\lambda} T\right) y^{\prime}$ by $y(x)$. 
Note that the coefficients of $y^{\prime \prime}, y^{\prime}$, and $y$ in (5) are polynomials of degree $n+1, n$, and at most $n-1$, respectively.

Theorem 3.2 Let $y$ represent an $s l_{2}$ critical point associated to $\left(\Lambda, z ; \Lambda_{\infty}, \lambda\right)$. Then equation (5) has a solution of the form $x^{\lambda+1} \tilde{y}(x)$ where $\tilde{y}(x)$ is a polynomial. If $\lambda$ is not a negative integer, then such a polynomial $\tilde{y}(x)$ is unique.

Moreover, there exists a finite set $C\left(\Lambda, z ; \Lambda_{\infty}\right) \subset \mathbb{C}$ such that if $\lambda$ is not a negative integer, $\lambda \notin C\left(\Lambda, z ; \Lambda_{\infty}\right)$, and y represents an $s l_{2}$ critical point associated to $\left(\Lambda, z ; \Lambda_{\infty}\right.$, $\lambda)$ then polynomial $\tilde{y}(x)$ represents an $s l_{2}$ critical point associated to $\left(\Lambda, z ;-\Lambda_{\infty}\right.$, $-\lambda-2)$.

We call $\tilde{y}(x)$ the immediate descendent of $y(x)$ with respect to $\lambda$.

Proof Equation (5) is a Fuchsian differential equation, with singular points at $0, z_{1}, \ldots$, $z_{n}, \infty$. At 0 the exponents of the equation are $0, \lambda+1$. Therefore, around 0 there is a solution of the form $u(x)=x^{\lambda+1} \tilde{y}(x)$ where $\tilde{y}(x)$ is a function holomorphic and non-vanishing at $x=0$. Such a solution is unique if $\lambda+1 \notin \mathbb{Z}_{\leq 0}$.

At a point $z_{s}$ the exponents are $\left(0, \Lambda_{s}+1\right)$. Since $y\left(z_{s}\right) \neq 0$ and $y$ is a polynomial solution, there is no monodromy around $z_{s}$, and thus $\tilde{y}(x)$ is an entire function, $\mathrm{cf}$ Scherbak [16, Lemma 7]. The function $\tilde{y}(x)$ is a polynomial since equation (5) is Fuchsian.

Denote

$$
W(f, g)=f^{\prime} g-f g^{\prime}
$$

the Wronskian of functions $f$ and $g$. We have

$$
W\left(y, x^{\lambda+1} \tilde{y}(x)\right)=x^{\lambda} T(x) .
$$

Thus

$$
\operatorname{deg} y+\operatorname{deg} \tilde{y}=\sum_{s=1}^{n} \Lambda_{s} .
$$

The polynomial $\tilde{y}(x)$ satisfies the equation

$$
F(x) \tilde{y}^{\prime \prime}-F(x) \ln ^{\prime}\left(x^{-\lambda-2} T(x)\right) \tilde{y}^{\prime}(x)+\tilde{C}(x) \tilde{y}(x)=0 .
$$

Thus if $\tilde{y}(x)$ is off-diagonal, then the polynomial $\tilde{y}(x)$ represents a critical point associated to $\left(\Lambda, z ;-\Lambda_{\infty},-\lambda-2\right)$.

Finally, we prove that for all but finitely many $\lambda$, the polynomial $\tilde{y}(x)$ is off-diagonal. If $\tilde{y}(x)$ is not off-diagonal, then $\tilde{y}(x)$ has zero of order $\Lambda_{s}+1$ at at least one of $z_{s}$. We show that such a pair $(y(x), \tilde{y}(x))$ is possible for at most finitely many $\lambda$. 
Consider a family of polynomials $y_{\lambda}(x)=\prod_{j}^{l}\left(x-t_{j, \lambda}\right)$ which algebraically depends on $\lambda$ and such that $y_{\lambda}(x)$ represents a critical point corresponding to $\left(\Lambda_{\infty}, \lambda\right)$ for all but finitely many $\lambda$.

We have a finite number of such families and for all but finitely many $\lambda$ every polynomial representing a critical point belongs to such a family.

Let $\tilde{y}_{\lambda}(x)=\prod_{j=1}^{\tilde{l}}\left(x-\tilde{t}_{j, \lambda}\right)$ be the descendent polynomial of $y_{\lambda}(x)$.

From (6) we get

$$
\frac{\lambda+1}{x}+\sum_{i=1}^{\tilde{l}} \frac{1}{x-\tilde{t}_{j, \lambda}}-\sum_{i=1}^{l} \frac{1}{x-t_{j, \lambda}}=\frac{(\lambda+\tilde{l}-l) \prod_{j=1}^{n}\left(x-z_{j}\right)^{\Lambda_{j}}}{x \prod_{i=1}^{\tilde{l}}\left(x-\tilde{t}_{j, \lambda}\right) \prod_{i=1}^{l}\left(x-t_{j, \lambda}\right)} .
$$

Let $\lambda$ tend to infinity. Comparing the main terms of asymptotics of the left and right hand sides, we conclude that the limit of $y_{\lambda} \tilde{y}_{\lambda}$ is $T$. The polynomial $T$ has zero of order $\Lambda_{s}$ at $z_{s}$ and therefore $\tilde{y}_{\lambda}$ cannot have a zero of order $\Lambda_{s}+1$ at $z_{s}$ for all but finitely many $\lambda$. It follows that $\tilde{y}_{\lambda}$ has a zero at $z_{s}$ for only finitely many values of $\lambda$.

Corollary 3.3 A polynomial $y(x)$ represents an $s l_{2}$ critical point associated to $\left(\Lambda_{\infty}, \lambda\right)$ if and only if $\operatorname{deg} y(x)=l, y(x)$ is off-diagonal with respect to $(\Lambda, z)$ and there exists a polynomial $\tilde{y}(x)$ such that $W\left(y, x^{\lambda+1} \tilde{y}\right)=x^{\lambda} T(x)$.

Note that if $y(x)$ represents a critical point associated to $\left(\Lambda_{\infty}, \lambda\right)$ and if the descendent polynomial $\tilde{y}(x)$ is off-diagonal then $\tilde{y}(x)$ represents a critical point associated to $\left(w \Lambda_{\infty}, w \cdot \lambda\right)$, where $w \neq$ id is the generator of the $s l_{2}$ Weyl group.

The polynomials $y(x)$ and $\tilde{y}(x)$ may coincide (up to a multiplicative constant). For example, if there are no $z_{s}$, if $l=0$ and $y(x)=1$, then $\tilde{y}(x)=1 /(\lambda+1)$. The polynomials $y(x)$ and $\tilde{y}(x)$ are constant multiples of each other, but they represent critical points associated to different weights $\left(\Lambda_{\infty}=0, \lambda\right)$ and $\left(\Lambda_{\infty}=0,-\lambda-2\right)$, respectively.

Assume that $\lambda \in \mathbb{C}$ is not an integer. Then the unordered pair $\{(y(x), \lambda),(\tilde{y}(x),-\lambda-$ $2)\}$, is called the $s l_{2}$ population originated at $(y(x), \lambda)$. Here $y(x), \tilde{y}(x)$ are considered up to a multiplicative constant.

Lemma 3.4 Let $\lambda$ be not an integer. Let $P=\{(y(x), \lambda),(\tilde{y}(x),-\lambda-2)\}$ be the $s l_{2}$ population originated at $(y(x), \lambda)$. Assume that $\tilde{y}(x)$ is off-diagonal. Then $y(x)$ is a descendent polynomial of $\tilde{y}(x)$ with respect to $-\lambda-2$ and the population originated at $(\tilde{y}(x),-\lambda-2)$ coincides with $P$. 
Proof We have

$$
x^{-\lambda-2} T=\left(x^{-\lambda-1}\right)^{2} x^{\lambda} T=\left(x^{-\lambda-1}\right)^{2} W\left(y, x^{\lambda+1} \tilde{y}\right)=W\left(\tilde{y},-x^{-\lambda-1} y\right),
$$

cf the Wronskian identities in [9, Lemma 9.2].

\subsection{The populations in the case of a general Kac-Moody algebra}

Recall that we fixed a Kac-Moody algebra $\mathfrak{g}=\mathfrak{g}(A)$ of rank $r$, a non-negative integer $n \in \mathbb{Z}_{\geq 0}$, an $n$-tuple of $\mathfrak{g}$-weights $\Lambda=\left(\Lambda_{i}\right)_{i=1}^{n}, \Lambda_{i} \in \mathcal{P}^{+}$, and an $n$-tuple $z=\left(z_{i}\right)_{i=1}^{n} \in \mathbb{C}^{n}$ such that $z_{i} \neq 0$ and $z_{i} \neq z_{j}$ if $i \neq j$.

For $i=1, \ldots, r$, we set

$$
T_{i}(x)=\prod_{s=1}^{n}\left(x-z_{s}\right)^{\left\langle\Lambda_{s}, \alpha_{i}^{\vee}\right\rangle} .
$$

Given a set of numbers $t=\left(t_{j}^{(i)}\right)_{i=1, \ldots, r}^{j=1, \ldots, l_{i}}$, we represent it by the $r$-tuple of polynomials $y=\left(y_{1}, \ldots, y_{r}\right)$, where $y_{i}(x)=\prod_{j=1}^{l_{i}}\left(x-t_{j}^{(i)}\right), i=1, \ldots, r$. We are interested only in the roots of the polynomials $y_{i}$, therefore we make no distinction between tuples $\left(y_{1}, \ldots, y_{r}\right)$ and $\left(c_{1} y_{1}, \ldots, c_{r} y_{r}\right)$, where $c_{i}$ are non-zero constants.

An $r$-tuple of polynomials $y$ is called off-diagonal with respect to $(\Lambda, z)$ if its roots do not belong to the union of singular hyperplanes in (3). Namely $y$ is off-diagonal if for $i=1, \ldots, n$, all roots of polynomial $y_{i}$ are simple, non-zero, different from the roots of polynomials $y_{j}$ for all $j$ such that $\left(\alpha_{j}, \alpha_{i}\right) \neq 0$ and different from the roots of polynomial $T_{i}$.

Since $\Lambda, z$ are fixed, we often call $r$-tuples of polynomials $y$ which are off-diagonal with respect to $(\Lambda, z)$ simply off-diagonal.

An $r$-tuple of polynomials $y$ is called fertile in the $i$-th direction, $i \in\{1, \ldots, r\}$ with respect to $\lambda$ if there exists a polynomial $\tilde{y}_{i}$ such that

$$
W\left(y_{i}, x^{\left\langle\lambda+\rho, \alpha_{i}^{\vee}\right\rangle} \tilde{y}_{i}\right)=x^{\left\langle\lambda, \alpha_{i}^{\vee}\right\rangle} T_{i} \prod_{j, j \neq i} y_{j}^{-a_{i j}} .
$$

Then the tuple of polynomials $y^{(i)}:=\left(y_{1}, \ldots, \tilde{y}_{i}, \ldots, y_{r}\right)$ is called an immediate descendent of $y$ in the $i$-th direction with respect to $\lambda$.

Recall that $s_{i} \in \mathcal{W}$ are elementary reflections in the Weyl group of $\mathfrak{g}$.

We call an $r$-tuple of polynomials $y^{\left(i_{1}, i_{2}, \ldots, i_{m}\right)}$, where $i_{k} \in\{1, \ldots, r\}, k=1, \ldots, m, a$ descendent of $y$ with respect to $\lambda$ in the directions $\left(i_{1}, \ldots, i_{m}\right)$ if there exist $r$-tuples 
of polynomials $y^{\left(i_{1}, i_{2}, \ldots, i_{k}\right)}, k=1, \ldots, m-1$, such that for $k=1, \ldots, m$, the $r$-tuple $y^{\left(i_{1}, i_{2}, \ldots, i_{k}\right)}$ is an immediate descendent of $y^{\left(i_{1}, i_{2}, \ldots, i_{k-1}\right)}$ in the $i_{k}$-th direction with respect to $\left(s_{i_{k-1}} \ldots s_{i_{2}} s_{i_{1}}\right) \cdot \lambda$.

An $r$-tuple of polynomials $y$ is called fertile with respect to $\lambda$ if it is fertile in all directions $i=1, \ldots, r$.

An $r$-tuple of polynomials $y$ is called super-fertile with respect to $\lambda$ if it is fertile with respect to $\lambda$ and all descendents $y^{\left(i_{1}, i_{2}, \ldots, i_{k}\right)}$ of $y$ with respect to $\lambda$ are fertile with respect to $\left(s_{i_{k}} \ldots s_{i_{1}}\right) \cdot \lambda$.

Theorem 3.5 Let $\mathfrak{g}$ be a Kac-Moody algebra. An $r$-tuple $y$ represents a $\mathfrak{g}$ critical point associated to $\left(\Lambda, z ; \Lambda_{\infty}, \lambda\right)$ if and only if $\operatorname{deg} y_{i}=l_{i}, i=1, \ldots, r, y$ is off-diagonal with respect to $(\Lambda, z)$ and fertile with respect to $\lambda$. Moreover, if an immediate descendent of $y$ in the direction $i, y^{(i)}=\left(y_{1}, \ldots, \tilde{y}_{i}, \ldots, y_{r}\right)$, is off-diagonal with respect to $(\Lambda, z)$ then it represents a $\mathfrak{g}$ critical point associated to $\left(\Lambda, z ; s_{i} \Lambda_{\infty}, s_{i} \cdot \lambda\right)$.

Proof The first part of the theorem follows immediately from the case of $s l_{2}$, see Corollary 3.3.

To show the second part we show that roots of $y^{(i)}$ satisfy system (3), where $\lambda$ is changed to $s_{i} \cdot \lambda$. Let $\tilde{t}_{j}^{(i)}$ denote the roots of $\tilde{y}_{i}$.

The equations of system (3) corresponding to coordinates $\tilde{t}_{j}^{(i)}$ are satisfied by Theorem 3.2.

The equations of system (3) corresponding to coordinates $\tilde{t}_{j}^{(k)}$ such that $a_{k i}=0$, are satisfied because these equations are the same for $\tilde{y}$ and $y$.

For any $k$, such that $k \neq i$ and $a_{i k} \neq 0$, choose a root $t_{j}^{(k)}$ of the polynomial $y_{k}$. Setting $x=t_{j}^{(k)}$ in the $i$-th equation of (8), we get

$$
\sum_{s} \frac{1}{t_{j}^{(k)}-t_{s}^{(i)}}=\sum_{s} \frac{1}{t_{j}^{(k)}-\tilde{t}_{s}^{(i)}}+\frac{\left\langle\lambda+\rho, \alpha_{i}^{\vee}\right\rangle}{t_{j}^{(k)}} .
$$

This implies that the equation of system (3) corresponding to the coordinate $\tilde{t}_{j}^{(k)}$ is satisfied as well.

Note that if the tuple $y^{(i)}$ is off-diagonal, then it is again fertile and we can find the $r$-tuple of polynomials $y^{(i, j)}$. However, in general, we do not know if the tuple $y^{(i)}$ is off-diagonal. It is true in the case of $s l_{2}$ and almost all non-integral weights $\lambda$, see Theorem 3.2. We have the following conjecture. 
Conjecture 3.6 An off-diagonal fertile tuple is super-fertile.

We prove this conjecture for the simple Lie algebras of types $A_{r}, B_{r}$, see Theorems 4.5 and 4.8.

For an $r$-tuple of polynomials $y$ and a $\mathfrak{g}$ weight $\lambda$, let $P(y, \lambda)$ be the set of all pairs of the form $\left(y^{\left(i_{1}, i_{2}, \ldots, i_{m}\right)},\left(s_{i_{m}} \ldots s_{i_{2}} s_{i_{1}}\right) \cdot \lambda\right)$, where $i_{k} \in\{1, \ldots, r\}, m \in \mathbb{Z}_{\geq 0}, k=1, \ldots, m$, and $y^{\left(i_{1}, i_{2}, \ldots, i_{m}\right)}$ is a descendent of $y$ with respect to $\lambda$ in directions $\left(i_{1}, \ldots, i_{m}\right)$.

We call the set $P(y, \lambda)$ the prepopulation originated at $(y, \lambda)$. If $y$ is a super-fertile $r$-tuple with respect to $\lambda$ then we call the set $P(y, \lambda)$ the population originated at $(y, \lambda)$.

Lemma 3.7 Let $y$ be super-fertile with respect to $\lambda$ and let $P$ be the population originated at $(y, \lambda)$. Let $(\tilde{y}, \tilde{\lambda}) \in P$. Then $\tilde{y}$ is super-fertile with respect to $\tilde{\lambda}$ and the population originated at $(\tilde{y}, \tilde{\lambda})$ is also $P$. In particular, different populations do not intersect.

Proof By Lemma 3.4 we obtain that if $y^{(i)}$ is an immediate descendent of $y$ in the direction $i$, then $y$ is also an immediate descendent of $y^{(i)}$ in the direction $i$. The lemma follows.

We call a weight $\lambda$ strongly non-integral if for any element of the Weyl group $s \in \mathcal{W}$ and any $i \in\{1, \ldots, r\}$ the number $\left\langle s \cdot \lambda, \alpha_{i}^{\vee}\right\rangle$ is not an integer.

Note that if $\lambda$ is strongly non-integral, then, in particular, the weights $s \cdot \lambda, s \in \mathcal{W}$, do not belong to the reflection hyperplanes. Therefore the map $\mathcal{W} \rightarrow \mathcal{W} \cdot \lambda$ mapping $w \in \mathcal{W}$ to $w \cdot \lambda$ is bijective.

Lemma 3.8 Let $\lambda$ be strongly non-integral and let $r$-tuples $y_{1}, y_{2}$ be descendents of an $r$-tuple $y$ with respect to $\lambda$ in the directions $\left(i_{1}, \ldots, i_{m}\right)$. Then the $r$-tuples $y_{1}, y_{2}$ coincide.

Proof Lemma follows from the corresponding $s l_{2}$ statement, see Theorem 3.2.

Let $\lambda$ be strongly non-integral. Let $y$ be super-fertile with respect to $\lambda$. Let $P$ be the population originated at $(y, \lambda)$. For $i \in\{1, \ldots, r\}$, let $a_{i}: P \rightarrow P$ be the map of the simple reproduction in the $i$-th direction which maps $(\tilde{y}, \tilde{\lambda})$ to $\left(\tilde{y}^{(i)}, s_{i} \cdot \tilde{\lambda}\right)$. According to Lemma 3.8, the map $a_{i}$ is well defined. By Lemma 3.4 we have $a_{i}^{2}=i d$. In particular $a_{i}$ are invertible.

Let $\mathcal{A}$ be the subgroup of the group of all permutations of the elements in $P$ generated by $a_{i}, i=1, \ldots, r$. 
Conjecture 3.9 There is an isomorphism of groups $\mathcal{A} \rightarrow \mathcal{W}$ which maps $a_{i}$ to $s_{i}$.

If $(\tilde{y}, \tilde{\lambda}) \in P$ then $\tilde{y}$ is a descendent of $y$ in some directions $\left(i_{1}, \ldots, i_{m}\right)$ and we have $\tilde{\lambda}=w \cdot \lambda$ for some $w \in \mathcal{W}$. Since $\lambda$ is strongly non-integral, such $w$ is unique and we have $w=s_{i_{m}} \ldots s_{i_{1}}$. This defines a map

$$
\tau: \quad P \quad \rightarrow \mathcal{W}
$$

Since $y$ is super-fertile, $\tau$ is a surjective map. Conjecture 3.9 is true if and only if the map $\tau$ is a bijection for all populations $P$.

Note that the map $\tau=\tau(y, \lambda)$ depends on the choice of the element $(y, \lambda) \in P$. However, if it is bijective for one element of the population, then it is clearly bijective for all elements of this population.

Conjecture 3.9 for the case of $s l_{2}$ is proved in Lemma 3.4.

Below we prove Conjecture 3.9 for simple Lie algebras.

\section{Proof of Conjecture 3.9 for simple Lie algebras}

\subsection{The case of $s l_{N+1}$}

We have roots $\alpha_{1}, \ldots, \alpha_{N}$ with scalar products $\left(\alpha_{i}, \alpha_{i}\right)=2,\left(\alpha_{i}, \alpha_{i \pm 1}\right)=-1$ and 0 otherwise.

We fix weights $\Lambda=\left(\Lambda_{1}, \ldots, \Lambda_{n}\right), \Lambda_{\infty}$, points $z=\left(z_{1}, \ldots, z_{n}\right)$. The weights $\Lambda_{s}$, $s=1, \ldots, n$, are dominant integral $s l_{N+1}$ weights and the points $z_{s}, s=1, \ldots, n$, are non-zero, pairwise different complex numbers. We define polynomials $T_{i}$ as in (7).

We also fix a strongly non-integral $s l_{N+1}$ weight $\lambda$.

For any $N$-tuple of functions $y=\left(y_{1}, \ldots, y_{N}\right)$ and $s l_{N+1}$ weight $\lambda$, we set $y_{N+1}=1$ and define the linear differential operator of order $N+1$ :

$$
\begin{aligned}
D(y, \lambda) & =\left(\partial-\ln ^{\prime}\left(\frac{\prod_{s=1}^{N} x^{\left(\lambda, \alpha_{s}\right)} T_{s}}{y_{N}}\right)\right) \ldots\left(\partial-\ln ^{\prime}\left(\frac{y_{2} T_{1} x^{\left(\lambda, \alpha_{1}\right)}}{y_{1}}\right)\right)\left(\partial-\ln ^{\prime}\left(y_{1}\right)\right) \\
& =\prod_{i}^{0 \rightarrow N}\left(\partial-\ln ^{\prime}\left(\frac{y_{N+1-i} \prod_{s=1}^{N-i} x^{\left(\lambda, \alpha_{s}\right)} T_{s}}{y_{N-i}}\right)\right) .
\end{aligned}
$$

For an $N$-tuple of polynomials $y$ and an $s l_{N+1}$ weight $\lambda$, let as before the prepopulation $P=P(y, \lambda)$ be the set of all descendents of $y$ paired with the corresponding weight. 
Lemma 4.1 Let $y$ represent an $s l_{N+1}$ critical point associated to $\left(\Lambda_{\infty}, \lambda\right)$. Then the prepopulation $P$ contains the elements

$$
\left(y^{(i, i-1, \ldots, 1)},\left(s_{i} s_{i-1} \ldots s_{1}\right) \cdot \lambda\right),
$$

where $i=0, \ldots, N$.

Proof Since $y$ represents a critical point, it is fertile and none of $y_{j}$ has multiple roots. Moreover we have the Bethe Ansatz equation (3) for each root of each polynomial $y_{j}$.

In particular there exist polynomials $\tilde{y}_{i}$ such that

$$
W\left(y_{i}, x^{\left(\lambda+\rho, \alpha_{i}\right)} \tilde{y}_{i}\right)=x^{\left(\lambda, \alpha_{i}\right)} y_{i-1} y_{i+1} T_{i} .
$$

Note that if $t_{j}^{(i-1)}$ is a root of $y_{i-1}$ then either $\tilde{y}_{i}\left(t_{j}^{(i-1)}\right) \neq 0$ or $\tilde{y}_{i}$ vanishes at $t_{j}^{(i-1)}$ to order 2. In the former case, in the same way as in Theorem 3.5, we see that the Bethe Ansatz equation for the root $t_{j}^{(i-1)}$ of $y_{i-1}$ in the $N$-tuple $y^{(i)}$ is still valid. In addition the Bethe Ansatz equations for roots of $y_{1}, \ldots, y_{i-2}$ in the $N$-tuple $y^{(i)}$ are also satisfied since they are exactly the same as in the $N$-tuple $y$.

Consider the next equation for $\tilde{y}_{i-1}$ :

$$
W\left(y_{i-1}, x^{\left(s_{i} \cdot \lambda+\rho, \alpha_{i-1}\right)} \tilde{y}_{i-1}\right)=x^{\left(s_{i} \cdot \lambda, \alpha_{i-1}\right)} y_{i-2} \tilde{y}_{i} T_{i-1} .
$$

We have

$$
x^{\left(s_{i} \cdot \lambda+\rho, \alpha_{i-1}\right)} \tilde{y}_{i-1}=y_{i-1} \int \frac{x^{\left(s_{i} \cdot \lambda, \alpha_{i-1}\right)} y_{i-2} \tilde{y}_{i} T_{i-1}}{y_{i-1}^{2}} d x .
$$

We claim that the integrand does not have residues. Indeed, the residues could occur only at the roots $t_{j}^{(i-1)}$ of $y_{i-1}$. If $\tilde{y}_{i}\left(t_{j}^{(i-1)}\right)=0$ then the integrand is holomorphic at $x=t_{j}^{(i-1)}$. If $\tilde{y}_{i}\left(t_{j}^{(i-1)}\right) \neq 0$ then the absence of the residue is equivalent to the Bethe Ansatz equation corresponding to $t_{j}^{(i-1)}$ which is satisfied. Therefore $\tilde{y}_{i-1}$ is a polynomial and there exists a descendent $y^{(i, i-1)}$.

Similarly, we prove that $y^{(i, i-1, \ldots, i-m)}$ is a well-defined $N$-tuple of polynomials for $m=2, \ldots, i-1$.

If $(\tilde{y}, \tilde{\lambda}) \in P$ then we write

$$
\tilde{\lambda}=w(\tilde{\lambda}) \cdot \lambda=\lambda-\sum_{i=1}^{N} a_{i}(\tilde{\lambda}) \alpha_{i},
$$

where $w(\tilde{\lambda}) \in \mathcal{W}$ and $a_{i}(\tilde{\lambda}) \in \mathbb{R}$. Define the shifted prepopulation $\bar{P}(y, \lambda)$ as the following set of $N$-tuples of functions:

$$
\bar{P}=\left\{\left(x^{a_{1}(\tilde{\lambda})} \tilde{y}_{1}, x^{a_{2}(\tilde{\lambda})} \tilde{y}_{2}, \ldots, x^{a_{N}(\tilde{\lambda})} \tilde{y}_{N}\right) \mid(\tilde{y}, \tilde{\lambda}) \in P\right\} .
$$


Note that the shifted prepopulation $\bar{P}(y, \lambda)$ depends on the choice of an element $(y, \lambda) \in P$. However, the difference is not very essential: if $(\tilde{y}, \tilde{\lambda}) \in P$ and $\bar{P}(\tilde{y}, \tilde{\lambda})$ is the corresponding shifted prepopulation then there exists $a_{i} \in \mathbb{R}$ such that the $i$-th function in any $N$-tuples in $\bar{P}(\tilde{y}, \tilde{\lambda})$ is obtained via multiplication of the $i$-th function of the corresponding $N$-tuple in $\bar{P}(y, \lambda)$ by $x^{a_{i}}$.

Lemma 4.2 Let $\tilde{y} \in \bar{P}(y, \lambda)$. Then $D(\tilde{y}, \lambda)=D(y, \lambda)$.

Proof Let $(v, \mu) \in P$ and let $\left(v^{(i)}, s_{i} \cdot \mu\right) \in P$ be the immediate descendent of $v$ with respect to $\mu$ in the direction $i$. Then we have $v_{k}^{(i)}=v_{k}$ for $k \neq i$ and $W\left(v_{i}, v_{i}^{(i)} x^{\left(\mu+\rho, \alpha_{i}\right)}\right)=x^{\left(\mu, \alpha_{i}\right)} v_{i-1} v_{i+1} T_{i}$. The last relation can be rewritten as

$$
W\left(x^{a_{i}(\mu)} v_{i}, v_{i}^{(i)} x^{a_{i}\left(s_{i} \cdot \mu\right)}\right)=x^{\left(\lambda, \alpha_{i}\right)} v_{i-1} x^{a_{i-1}(\mu)} v_{i+1} x^{a_{i+1}(\mu)} T_{i} .
$$

Let

$$
\begin{aligned}
(\bar{v}, \mu) & =\left(\left(x^{a_{1}(\mu)} v_{1}, \ldots, x^{a_{N}(\mu)} v_{N}\right), \mu\right) \in \bar{P}(y, \lambda), \\
\left(\bar{v}^{(i)}, s_{i} \cdot \mu\right) & =\left(\left(x^{a_{1}\left(s_{i} \cdot \mu\right)} v_{1}^{(i)}, \ldots, x^{a_{N}\left(s_{i} \cdot \mu\right)} v_{N}^{(i)}\right), s_{i} \cdot \mu\right) \in \bar{P}(y, \lambda) .
\end{aligned}
$$

Identity (11) reads $W\left(\bar{v}_{i}, \bar{v}_{i}^{(i)}\right)=x^{\left(\lambda, \alpha_{i}\right)} \bar{v}_{i-1} \bar{v}_{i+1} T_{i}$ and therefore

$$
\begin{gathered}
-\ln ^{\prime \prime}\left(\bar{v}_{i}\right)+\ln ^{\prime}\left(\bar{v}_{i}\right)\left(\ln ^{\prime}\left(T_{i} x^{\left(\lambda, \alpha_{i}\right)} \bar{v}_{i-1} \bar{v}_{i+1}\right)-\ln ^{\prime}\left(\bar{v}_{i}\right)\right)= \\
-\ln ^{\prime \prime}\left(\bar{v}_{i}^{(i)}\right)+\ln ^{\prime}\left(\bar{v}_{i}^{(i)}\right)\left(\ln ^{\prime}\left(T_{i} x^{\left(\lambda, \alpha_{i}\right)} \bar{v}_{i-1}^{(i)} \bar{v}_{i+1}^{(i)}\right)-\ln ^{\prime}\left(\bar{v}_{i}^{(i)}\right)\right) .
\end{gathered}
$$

Compare $D(\bar{v})$ with $D\left(\bar{v}^{(i)}\right)$. All factors but two successive ones in these operators are the same. The products of the two middle factors are the same by (12)

We call $D(y, \lambda)=D$ the operator associated to the shifted prepopulation $\bar{P}(y, \lambda)$. It follows from Lemma 4.2 if $\tilde{y} \in \bar{P}(y, \lambda)$ then $D \tilde{y}_{1}=0$.

We use the following notation for Wronskians and divided Wronskians:

$$
\begin{aligned}
W\left(u_{1}, \ldots, u_{i}\right) & =\operatorname{det}\left(u_{k}^{(j-1)}\right)_{k, j=1}^{i}, \\
W^{\dagger}\left(u_{1}, \ldots, u_{i}\right) & =\frac{W\left(u_{1}, \ldots, u_{i}\right)}{\left(x^{\left(\lambda, \alpha_{1}\right)} T_{1}\right)^{i-1}\left(x^{\left(\lambda, \alpha_{2}\right)} T_{2}\right)^{i-2} \ldots\left(x^{\left(\lambda, \alpha_{i-1}\right)} T_{i-1}\right)},
\end{aligned}
$$

$i=1, \ldots, N+1$, where $u_{k}^{(j-1)}$ denotes the $(j-1)$ st derivative of $u_{k}$ with respect to variable $x$.

Lemma 4.3 Let $y$ either represent an $s l_{N+1}$ critical point associated to $\left(\Lambda_{\infty}, \lambda\right)$ or be super-fertile with respect to $\lambda$. Then there exist functions $u_{1}, \ldots, u_{N+1}$ such that $D u_{i}=0, y_{i}=W^{\dagger}\left(u_{1}, \ldots, u_{i}\right)$ for $i=1, \ldots, N+1$ and $u_{i} x^{-\left(\lambda+\rho, \alpha_{1}+\cdots+\alpha_{i-1}\right)}$ are polynomials for $i=1, \ldots, N+1$. 
Proof Let $u_{i}$ to be the first coordinate of the $N$-tuple in the element of $\bar{P}(y, \lambda)$ corresponding to $y^{(i-1, i-2, \ldots, 1)}$ which is a descendent of $y$ with respect to $\lambda$ in directions $(i-1, i-2, \ldots, 1)$. If $y$ represents an $s l_{N+1}$ critical point with respect to $\lambda$ then such an $N$-tuple exists by Lemma 4.1 .

We have $D u_{i}=0$ by Lemma 4.2.

The condition $W^{\dagger}\left(u_{1}, \ldots, u_{i}\right)=y_{i}$ follows from the standard Wronskian identities, cf [9, proof of Lemma 5.5].

Corollary 4.4 Let $y$ be an $N$-tuple of polynomials and $l_{i}=\operatorname{deg} y_{i}, i=1, \ldots, N$. Let $\Lambda_{\infty}$ be given by (1). Let $y$ represent an $s l_{N+1}$ critical point associated to $\left(\Lambda_{\infty}, \lambda\right)$ or let $y$ be super-fertile with respect to $\lambda$. Then the kernel of the operator $D(y, \lambda)$ is spanned by functions of the form

$$
p_{0}, p_{1} x^{\left(\lambda+\rho, \alpha_{1}\right)}, \ldots, p_{N} x^{\left(\lambda+\rho, \alpha_{1}+\cdots+\alpha_{N}\right)},
$$

where $p_{i}$ is a polynomial of degree $\operatorname{deg} y_{1}+\left(\Lambda_{\infty}, \alpha_{1}+\cdots+\alpha_{i}\right), p_{i}(0) \neq 0$. The only singular points of the operator $D(y, \lambda)$ in $\mathbb{C}^{*}$ are regular singular points located at $z_{1}, \ldots, z_{n}$, and the exponents at $z_{i}, i=1, \ldots, n$, are

$$
z_{i}: 0,\left(\Lambda_{i}+\rho, \alpha_{1}\right),\left(\Lambda_{i}+\rho, \alpha_{1}+\alpha_{2}\right), \ldots,\left(\Lambda_{i}+\rho, \alpha_{1}+\cdots+\alpha_{N}\right),
$$

Conversely, if a linear differential operator $D$ of order $N+1$ has the kernel spanned by functions of the form (13) and the only non-zero singular points of $D$ in $\mathbb{C}^{*}$ are regular singular points at $z_{1}, \ldots, z_{n}$ with the exponents given by (14), then the $N$-tuple $y$ given by the divided Wronskians

$$
y_{i}=W^{\dagger}\left(p_{0}, p_{1} x^{\left(\lambda+\rho, \alpha_{1}\right)}, \ldots, p_{i-1} x^{\left(\lambda+\rho, \sum_{j=1}^{i-1} \alpha_{j}\right)}\right)
$$

is super-fertile with respect to $\lambda$ and satisfies $\operatorname{deg} y_{i}=l_{i}, i=1, \ldots, N$.

Proof For $i=0, \ldots, N$, we set

$$
p_{i}=u_{i+1} x^{-\left(\lambda+\rho, \alpha_{1}+\cdots+\alpha_{i}\right)},
$$

where $u_{1}, \ldots, u_{N+1}$ are as in Lemma 4.3. By Lemma 4.3, $p_{0}, \ldots, p_{N}$ are polynomials.

Now, the first part of Corollary 4.4 follows from Lemma 4.3 by standard Wronskian identities, cf [9, Lemmas 5.8 and 5.10].

Conversely, let $V$ be the kernel of the operator $D$. We have $(N+1)$ ! distinguished full flags in $V$ such that the divided Wronskians of all spaces which form the flags are of the form $x^{a} p(x)$ where $p(x)$ is a polynomial. Namely, for a permutation $w \in S_{N+1}$ of the 
set $\{0,1, \ldots, N\}$ we have a full flag $\mathcal{F}_{w}$ such that the space of dimension $i$ is spanned by $p_{w(0)} x^{(\lambda+\rho), \alpha_{w(0)}} \ldots, p_{w(i-1)} x^{(\lambda+\rho), \alpha_{w(i-1)}}$.

For each such flag $\mathcal{F}_{w}$, we have the corresponding element $p_{w}$ in $\bar{P}(y, \lambda)$. Each element $p_{w}$ is obviously fertile and the immediate descendents of $p_{w}$ in the $i$-th direction is $p_{(i, i+1) w}$. Therefore, the $N$-tuple $y$ is fertile with respect to $\lambda$.

Theorem 4.5 Conjectures 3.6, 3.9 hold for the case of $s l_{N+1}$.

Proof Conjecture 3.6 follows from Theorem 3.5 and Corollary 4.4.

Conjecture 3.9 in the case of $s l_{N+1}$ follows from the proof of the converse statement of Corollary 4.4.

\subsection{The case of $B_{2}$}

In the case of $B_{2}$ we have two roots $\alpha_{1}, \alpha_{2}$ such that $\left(\alpha_{1}, \alpha_{1}\right)=4,\left(\alpha_{2}, \alpha_{2}\right)=2$, $\left(\alpha_{1}, \alpha_{2}\right)=-2$.

The key observation is that $B_{2}$ populations can be embedded in $s l_{4}$ populations.

Given a $B_{2}$ weight $\Lambda$, define the $s l_{4}$ weight $\Lambda^{A}$ by

$$
\left\langle\Lambda^{A},\left(\alpha_{1}^{A}\right)^{\vee}\right\rangle=\left\langle\Lambda^{A},\left(\alpha_{3}^{A}\right)^{\vee}\right\rangle=\left\langle\Lambda, \alpha_{1}^{\vee}\right\rangle, \quad\left\langle\Lambda^{A},\left(\alpha_{2}^{A}\right)^{\vee}\right\rangle=\left\langle\Lambda, \alpha_{2}^{\vee}\right\rangle,
$$

where $\alpha_{i}^{A}$ are roots of $s l_{4}$.

Note that if $\Lambda$ is strongly non-integral then $\Lambda^{A}$ is strongly non-integral.

Lemma 4.6 A pair $y=\left(y_{1}, y_{2}\right)$ represents a $B_{2}$ critical point associated to $(z, \Lambda$; $\left.\Lambda_{\infty}, \lambda\right)$ if and only if the triple $y^{A}=\left(y_{1}, y_{2}, y_{1}\right)$ represents an $s l_{4}$ critical point associated to $\left(z, \Lambda^{A} ; \Lambda_{\infty}^{A}, \lambda^{A}\right)$. Moreover there is an embedding $P(y, \lambda) \rightarrow P^{A}\left(y^{A}, \lambda^{A}\right)$ which sends $\left(\left(\tilde{y}_{1}, \tilde{y}_{2}\right), \tilde{\lambda}\right) \in P(y, \lambda)$ to $\left(\left(\tilde{y}_{1}, \tilde{y}_{2}, \tilde{y}_{1}\right), \tilde{\lambda}^{A}\right) \in P^{A}\left(y^{A}, \lambda^{A}\right)$.

Proof Follows immediately from the definitions.

Theorem 4.7 Conjecture 3.9 holds in the case of root system $B_{2}$.

Proof Recall the surjective maps $\tau: P \rightarrow \mathcal{W}$ and $\tau^{A}: P^{A} \rightarrow \mathcal{W}^{A}$, where $\mathcal{W}$ and $\mathcal{W}^{A}$ are the $B_{2}$ and $A_{3}$ Weyl groups, see (9). Then we clearly have $(\tau(y) \cdot \lambda)^{A}=\tau^{A}\left(y^{A}\right) \cdot \lambda^{A}$. By Theorem 4.5, the map $\tau^{A}$ is injective.

Therefore $\tau$ is injective and hence bijective. 
Theorem 4.8 Conjecture 3.6 holds in the case of simple Lie algebras of type $B_{N}$.

Proof Similarly to the case $N=2$, the $N$-tuple $\left(y_{1}, \ldots, y_{N}\right)$ represents a critical point of type $B_{N}$ if and only if the $(2 N-1)$-tuple $\left(y_{1}, \ldots, y_{N-1}, y_{N}, y_{N-1}, \ldots, y_{1}\right)$ represents a critical point of type $A_{2 N-1}$, see also [9]. The tuple $\left(y_{1}, \ldots, y_{N}\right)$ is super-fertile in $B_{N}$ sense because the $(2 N-1)$-tuple $\left(y_{1}, \ldots, y_{N-1}, y_{N}, y_{N-1}, \ldots, y_{1}\right)$ is super-fertile in $A_{2 N-1}$ sense.

\subsection{The case of the root systems of types $B, C, D, E$ and $F$}

From the $s l_{2}, s l_{3}$ and $B_{2}$ cases, we obtain the general case (except for $G_{2}$ ).

Theorem 4.9 Conjecture 3.9 holds in the case of the root systems of types $B, C, D, E$ and $F$.

Proof Let $\mathfrak{g}$ be a rank $r$ simple Lie algebra of type $B, C, D, E$ or $F$.

The Weyl group of $\mathfrak{g}$ is a finite group described by the generators and relations:

$$
\mathcal{W}=<s_{1}, \ldots, s_{r}>/\left(s_{i}^{2}=\left(s_{i} s_{j}\right)^{-\left(\alpha_{i}, \alpha_{j}\right)+2}=1, i, j=1, \ldots, r, i \neq j\right) .
$$

Here $<s_{1}, \ldots, s_{r}>$ denotes the free group with generators $s_{1}, \ldots, s_{r}$.

Note that in our case $\left(\alpha_{i}, \alpha_{j}\right)$ takes values $2,0,-1$ or -2 .

Let $\lambda$ be strongly non-integral, $y$ represent a $\mathfrak{g}$-critical point associated to $\left(\Lambda_{\infty}, \lambda\right)$ and let $P=P(y, \lambda)$ be the corresponding population.

We have the corresponding relations in the populations among descendents:

- $y^{(i, i)}=y$,

- $y^{(i, j)}=y^{(j, i)}$ if $\left(\alpha_{i}, \alpha_{j}\right)=0$,

- $y^{(i, j, i)}=y^{(j, i, j)}$ if $\left(\alpha_{i}, \alpha_{j}\right)=-1$,

- $y^{(i, j, i, j)}=y^{(j, i, j, i)}$ if $\left(\alpha_{i}, \alpha_{j}\right)=-2$.

The first relation follows from the case of $s l_{2}$, Lemma 3.4, the second relation is obvious, the third relation follows from the case of $s l_{3}$, Theorem 4.5, and the fourth relation follows from the case of $B_{2}$, Theorem 4.7.

Therefore, $P$ has at most $|\mathcal{W}|$ elements. 


\subsection{The case of $G_{2}$}

In the case of $G_{2}$ we have two roots $\alpha_{1}, \alpha_{2}$ such that $\left(\alpha_{1}, \alpha_{1}\right)=2,\left(\alpha_{2}, \alpha_{2}\right)=6$, $\left(\alpha_{1}, \alpha_{2}\right)=-3$.

The key observation is that $G_{2}$ populations can be embedded in $C_{3}$ populations.

Given a $G_{2}$ weight $\Lambda$, define the $C_{3}$ weight $\Lambda^{C}$ by

$$
\left\langle\Lambda^{C},\left(\alpha_{1}^{C}\right)^{\vee}\right\rangle=\left\langle\Lambda^{C},\left(\alpha_{3}^{C}\right)^{\vee}\right\rangle=\left\langle\Lambda, \alpha_{1}^{\vee}\right\rangle, \quad\left\langle\Lambda^{C},\left(\alpha_{2}^{C}\right)^{\vee}\right\rangle=\left\langle\Lambda, \alpha_{2}^{\vee}\right\rangle,
$$

where $\alpha_{i}^{C}$ are roots of $C_{3}$.

Note that if $\Lambda$ is strongly non-integral then $\Lambda^{C}$ is strongly non-integral.

Lemma 4.10 A pair $y=\left(y_{1}, y_{2}\right)$ represents a $G_{2}$ critical point associated to $(\Lambda, z$; $\left.\Lambda_{\infty}, \lambda\right)$ if and only if the triple $y^{C}=\left(y_{1}, y_{2}, y_{1}\right)$ represents a $C_{3}$ critical point associated to $\left(\Lambda_{i}^{C}, z ; \Lambda_{\infty}^{C}, \lambda^{C}\right)$. Moreover there is an embedding $P(y, \lambda) \rightarrow P^{C}\left(y^{C}, \lambda^{C}\right)$ which sends $\left(\left(\tilde{y}_{1}, \tilde{y}_{2}\right), \tilde{\lambda}\right) \in P(y, \lambda)$ to $\left(\left(\tilde{y}_{1}, \tilde{y}_{2}, \tilde{y}_{1}\right), \tilde{\lambda}^{C}\right) \in P^{C}\left(y^{C}, \lambda^{C}\right)$.

Proof Follows immediately from definitions.

Theorem 4.11 Conjecture 3.9 holds in the case of the root system $G_{2}$.

Proof Recall the surjective maps $\tau: P \rightarrow \mathcal{W}$ and $\tau^{C}: P^{C} \rightarrow \mathcal{W}^{C}$, where $\mathcal{W}$ and $\mathcal{W}^{C}$ are the $G_{2}$ and $C_{3}$ Weyl groups, see (9). Then we clearly have $(\tau(y) \cdot \lambda)^{C}=\tau^{C}\left(y^{C}\right) \cdot \lambda^{C}$. By Theorem 4.9, the map $\tau^{C}$ is injective.

To summarize, we have

Corollary 4.12 Conjecture 3.9 holds in the case of all simple Lie algebras.

\section{Weyl group actions on Bethe vectors}

\subsection{The trigonometric Gaudin operators and Bethe vectors}

Let $\mathfrak{g}$ be a simple complex Lie algebra of rank $r$ with the Killing form $($,$) . We choose$ a Cartan subalgebra $\mathfrak{h}$, simple roots $\alpha_{i}, i=1, \ldots, r$. We identify $\mathfrak{h}$ with $\mathfrak{h}^{*}$ using the Killling form on $\mathfrak{g}$. Let $F_{i}, E_{i}, i=1, \ldots, r$, be the Chevalley generators of $\mathfrak{g}$. 
Let $\Delta$ be the root system, let $\Delta_{ \pm}$be the sets of positive and negative roots and let $\mathfrak{g}=\mathfrak{h} \oplus \sum_{\alpha \in \Delta} g_{\alpha}$ be the root decomposition. Let $e_{\alpha}, \alpha \in \Delta$, be generators of $g_{\alpha}$ such that $\left(e_{\alpha}, e_{-\alpha}\right)=1$. Let $\left\{h_{j}\right\}_{j=1, \ldots, r}$ be an orthonormal basis of the Cartan algebra $\mathfrak{h} \subset \mathfrak{g}$.

Set

$\Omega^{0}=\frac{1}{2} \sum_{j=1}^{r} h_{j} \otimes h_{j}, \quad \Omega^{+}=\Omega^{0}+\sum_{\alpha \in \Delta_{+}} e_{\alpha} \otimes e_{-\alpha}, \quad \Omega^{-}=\Omega^{0}+\sum_{\alpha \in \Delta_{+}} e_{-\alpha} \otimes e_{\alpha}$.

The trigonometric $R-$ matrix is defined by

$$
r(z)=\frac{\Omega^{+} z+\Omega^{-}}{z-1}
$$

We fix $z, \Lambda, \Lambda_{\infty}, l, \lambda$ as in Section 2.2. Let $L_{1}, \ldots, L_{n}$ be irreducible $\mathfrak{g}$-modules with highest weights $\Lambda_{1}, \ldots, \Lambda_{n}$ and let $V=L_{1} \otimes \cdots \otimes L_{n}$. Let $V[\mu] \subset V$ be the subspace of $V$ of all vectors of weight $\mu$.

We write $X^{(k)}$ for an operator $X \in \mathfrak{g}$ acting on the $k$-th factor. Similarly we write $X^{(k, l)}$ for an operator $X \in \mathfrak{g} \otimes \mathfrak{g}$ acting on the $k$-th and $l$-th factors.

The trigonometric Gaudin operators $H_{i}(\lambda), i=1, \ldots, n$, are defined by

$$
H_{i}(\lambda)=\lambda^{(i)}+\sum_{j=1, \ldots, n, j \neq i} r^{(i, j)}\left(z_{i} / z_{j}\right) .
$$

The trigonometric Gaudin operators depend on $\lambda \in \mathfrak{h}$ and act in $V$. The trigonometric Gaudin operators all commute, $\left[H_{i}(\lambda), H_{j}(\lambda)\right]=0, i, j=1, \ldots, n$. The trigonometric Gaudin operators commute with the action of $\mathfrak{h}$ on $V$ and therefore preserve every weight subspace of $V$.

For a given $\lambda$, common eigenvectors of the trigonometric Gaudin operators $H_{i}(\lambda)$ in the weight subspace $V\left[\Lambda_{\infty}\right]$ can be constructed by the Bethe Ansatz method as follows.

Let $l=l_{1}+\cdots+l_{n}$. Let $c$ be the unique non-decreasing function from $\{1, \ldots, l\}$ to $\{1, \ldots, r\}$ such that $\sharp c^{-1}(i)=l_{i}, i=1, \ldots, r$.

Let $P(l, n)$ be the set of sequences $I=\left(i_{1}^{1}, \ldots, i_{j_{1}}^{1} ; \ldots ; i_{1}^{n}, \ldots, i_{j_{n}}^{n}\right)$ of integers in $\{1, \ldots, r\}$ such that for all $i=1, \ldots, r$, the integer $i$ appears in $I$ precisely $l_{i}$ times. For $I \in P(l, n)$, and a permutation $\sigma \in S_{l}$ set $\sigma_{1}(i)=\sigma(i)$ for $i=1, \ldots, j_{1}$, and $\sigma_{s}(i)=\sigma\left(j_{1}+\cdots+j_{s-1}+i\right)$ for $s=2, \ldots, n$ and $i=1, \ldots, j_{s}$. Define

$$
S(I)=\left\{\sigma \in S_{l} \mid c\left(\sigma_{s}(j)\right)=i_{s}^{j} \text { for } s=1, \ldots, n \text { and } j=1, \ldots, j_{s}\right\} .
$$


For $I \in P(l, n)$ we define a vector in $V\left[\Lambda_{\infty}\right]$ by the formula

$$
F_{I} v=F_{i_{1}^{1}} \ldots F_{i_{j_{1}}^{1}} v_{1} \otimes \cdots \otimes F_{i_{1}^{n}} \ldots F_{i_{j_{n}}^{n}} v_{n}
$$

For $I \in P(l, n), \sigma \in S(I)$, we define a rational function of $t=\left(t_{i}^{(j)}\right)_{j=1, \ldots, r}^{i=1, \ldots, l_{j}}$ by the formula

$$
\omega_{I, \sigma}(t)=\omega_{\sigma_{1}(1), \ldots, \sigma_{1}\left(j_{1}\right)}\left(z_{1} ; t\right) \cdots \omega_{\sigma_{n}(1), \ldots, \sigma_{n}\left(j_{n}\right)}\left(z_{n} ; t\right),
$$

where

$$
\omega_{i_{1}, \ldots, i_{j}}(z ; t)=\frac{1}{\left(t_{i_{1}}-t_{i_{2}}\right) \cdots\left(t_{i_{j-1}}-t_{i_{j}}\right)\left(t_{i_{j}}-z\right)}
$$

and $\left(t_{1}, \ldots, t_{l}\right)=\left(t_{1}^{(1)}, \ldots, t_{l_{1}}^{(1)}, t_{1}^{(2)}, \ldots, t_{l_{2}}^{(2)}, \ldots, t_{1}^{(r)}, \ldots, t_{l_{r}}^{(r)}\right)$.

We define the weight function by

$$
\omega(t)=\sum_{I \in P(l, n)} \sum_{\sigma \in S(I)} \omega_{I, \sigma}(t) F_{I} v .
$$

The weight function $\omega(t)$ is a rational function of $t$ with values in $V\left[\Lambda_{\infty}\right]$.

Proposition 5.1 If $t$ is a critical point of the master function (2) associated to $\left(\Lambda_{\infty}, \lambda\right)$, then $\omega(t)$ is a well defined vector of weight $\Lambda_{\infty}$ in $V$ which is an eigenvector of the operators $H_{i}\left(\lambda+\rho+\Lambda_{\infty} / 2\right), i=1, \ldots, n$.

Proof Proposition 5.1 follows from the corresponding fact for the rational Gaudin operators, see [15] and the relation between rational and trigonometric Gaudin operators, see [5, Appendix B].

The vector $\omega(t)$ is called the Bethe vector associated to the critical point $t$. It is expected that for generic values of parameters, all critical points are non-degenerate and the Bethe vectors form a basis in $V$. In particular, the number of orbits of critical points and thus the number of populations should match the dimension of the subspace of all vectors of weight $\Lambda_{\infty}$ in $V$.

\subsection{Counting $s l_{N+1}$ critical points}

Let $L_{\Lambda}$ denote the irreducible $s l_{N+1}$ module of highest weight $\Lambda$.

Proposition 5.2 For almost all $\lambda$ the number of orbits of $s l_{N+1}$ critical points associated to $\left(\Lambda_{\infty}, \lambda\right)$ and counted with multiplicity does not exceed the dimension of the subspace of the weight $\Lambda_{\infty}$ in the tensor product $L_{\Lambda_{1}} \otimes \cdots \otimes L_{\Lambda_{n}}$. 
Proof By Lemma 2.1, for almost all $\lambda$, the number of critical points associated to $\left(\Lambda_{\infty}, \lambda\right)$ is finite. Therefore, there is a Zariski open set $O \subset \overline{\mathfrak{h}}^{*}$, such that the number of orbits of $s l_{N+1}$ critical points associated to $\left(\Lambda_{\infty}, \lambda\right)$ and counted with multiplicities is the same for all $\lambda \in O$.

If $\lambda$ is a dominant integral weight, then the number of orbits of critical points associated to $\left(\Lambda_{\infty}, \lambda\right)$ and counted with multiplicities is bounded from above by the multiplicity of $L_{\Lambda_{\infty}}$ in the tensor product $L_{\lambda} \otimes L_{\Lambda_{1}} \otimes \cdots \otimes L_{\Lambda_{n}}$, see $[9,1]$.

For any integer $M>0$, let $\mathcal{C}_{M}$ be the set of all weights $\lambda \in \mathfrak{h}^{*}$ such that the scalar products $\left(\lambda, \alpha_{i}\right)$ are integers greater than $M$.

If $\lambda \in \mathcal{C}_{M}$ and $M$ is large enough, then any singular vector of weight $\Lambda_{\infty}$ in the tensor product $L_{\lambda} \otimes L_{\Lambda_{1}} \otimes \cdots \otimes L_{\Lambda_{n}}$ is uniquely determined by its projection to $v_{\lambda} \otimes L_{\Lambda_{1}} \otimes \cdots \otimes L_{\Lambda_{n}}$, where $v_{\lambda}$ is the highest weight vector of $L_{\lambda}$. Therefore, the multiplicity of $L_{\Lambda_{\infty}}$ in the tensor product $L_{\lambda} \otimes L_{\Lambda_{1}} \otimes \cdots \otimes L_{\Lambda_{n}}$ equals the dimension of the subspace of weight $\Lambda_{\infty}$ in the tensor product $L_{\Lambda_{1}} \otimes \cdots \otimes L_{\Lambda_{n}}$.

For any $M \in \mathbb{Z}_{\geq 0}$, the set $\mathcal{C}_{M}$ is not contained in any proper algebraic subset in $\overline{\mathfrak{h}}^{*}$ and thus the proposition follows.

Proposition 5.3 For almost all $\lambda$ and almost all $\left(z_{1}, \ldots, z_{n}\right) \in \mathbb{C}^{n}$, all of the critical points are non-degenerate and the number of orbits of $s l_{2}$ critical points associated to $\left(\Lambda_{\infty}, \lambda\right)$ equals the dimension of the subspace of weight $\Lambda_{\infty}$ in the tensor product $L_{\Lambda_{1}} \otimes \cdots \otimes L_{\Lambda_{n}}$

Proof For almost all $\lambda$ the number of orbits of $s l_{2}$ critical points associated to $\left(\Lambda_{\infty}, \lambda\right)$ is the same.

If $\lambda$ is dominant integral, then the number of orbits of critical points associated to $\left(\Lambda_{\infty}, \lambda\right)$ for generic $z$ equals the multiplicity of $L_{\Lambda_{\infty}}$ in the tensor product $L_{\lambda} \otimes L_{\Lambda_{1}} \otimes \cdots \otimes L_{\Lambda_{n}}$ and all these orbits are non-degenerate, see [16, Theorem 1].

For dominant integral values of $\lambda$ which are large enough, the multiplicity of $L_{\Lambda_{\infty}}$ in the tensor product $L_{\lambda} \otimes L_{\Lambda_{1}} \otimes \cdots \otimes L_{\Lambda_{n}}$ equals the dimension of the subspace of weight $\Lambda_{\infty}$ in the tensor product $L_{\Lambda_{1}} \otimes \cdots \otimes L_{\Lambda_{n}}$.

In this case, almost all $\lambda$ means all but finitely many and therefore the proposition follows. 


\subsection{Actions of the Weyl group on Bethe vectors}

Let $\mathfrak{g}$ be a simple Lie algebra, $G$ the corresponding connected and simply connected Lie group. The group $G$ acts on any finite-dimensional irreducible representation of $\mathfrak{g}$. Let $\mathfrak{h} \subset \mathfrak{g}$ be a Cartan subalgebra, and $T \subset G$ the corresponding torus. The Weyl group of $\mathfrak{g}$ can be described as $N / T$ where $N=\left\{g \in G \mid g T g^{-1}=T\right\}$. In particular, this defines a projective action of the Weyl group on any tensor product of finite-dimensional irreducible representations of $\mathfrak{g}$. The projective action becomes an action in the zero weight subspace.

We fix our $z, \Lambda, \Lambda_{\infty}, l, \lambda$ as in Section 2.2. Let $L_{1}, \ldots, L_{n}$ be irreducible $\mathfrak{g}$-modules with highest weights $\Lambda_{1}, \ldots, \Lambda_{n}$ and let $V=L_{1} \otimes \cdots \otimes L_{n}$. Let $V[\mu]$ be the subspace of all vectors in $V$ of weight $\mu$. Let $P(V)=\{\mu, V[\mu] \neq 0\}$ be the set of all nontrivial weights in $V$.

We define the dynamical Weyl group acting on $V$ following [18].

Let $M_{\mu}$ denote the Verma module with highest weight $\mu, v_{\mu}$ a highest weight vector in $M_{\mu}$.

Let $M_{\mu}, M_{\lambda}$ be Verma modules. Two cases are possible:

a) $\operatorname{Hom}_{\mathfrak{g}}\left(M_{\mu}, M_{\lambda}\right)=0$ or

b) $\operatorname{Hom}_{\mathfrak{g}}\left(M_{\mu}, M_{\lambda}\right)=\mathbb{C}$ and every nontrivial homomorphism $M_{\mu} \rightarrow M_{\lambda}$ is an embedding.

Let $M_{\lambda}$ be a Verma module with dominant weight $\lambda \in P^{+}$. Then $\operatorname{Hom}_{\mathfrak{g}}\left(M_{\mu}, M_{\lambda}\right)=\mathbb{C}$ if and only if there is $w \in \mathcal{W}$ such that $\mu=w \cdot \lambda$.

Let $w=s_{i_{k}} \ldots s_{i_{1}}$ be a reduced presentation of an element of the Weyl group $\mathcal{W}$. Set $\alpha^{1}=\alpha_{i_{1}}$ and $\alpha^{j}=\left(s_{i_{1}} \ldots s_{i_{j-1}}\right)\left(\alpha_{i_{j}}\right)$ for $j=2, \ldots, k$. Let $n_{j}=\left(\lambda+\rho,\left(\alpha^{j}\right)^{\vee}\right)$. For a dominant $\lambda \in P^{+}$, the numbers $n_{j}$ are positive integers. Define a singular vector $v_{w \cdot \lambda}^{\lambda} \in M_{\lambda}$ by

$$
v_{w \cdot \lambda}^{\lambda}=\frac{\left(E_{-\alpha_{i_{k}}}\right)^{n_{k}}}{n_{1} !} \ldots \frac{\left(E_{-\alpha_{i_{1}}}\right)^{n_{1}}}{n_{k} !} v_{\lambda} .
$$

This vector does not depend on the reduced presentation, see [18].

For all $\lambda \in P^{+}, w \in \mathcal{W}$, fix an embedding $M_{w \cdot \lambda} \hookrightarrow M_{\lambda}$ sending $v_{w \cdot \lambda}$ to $v_{w \cdot \lambda}^{\lambda}$.

We say that $\lambda \in P^{+}$is generic with respect to $V$ if

- For any $\nu \in P(V)$ and any $v \in V[\nu]$, there exist a unique intertwining operator $\Phi_{\lambda}^{v}: M_{\lambda} \rightarrow M_{\lambda-\nu} \otimes V$ such that $\Phi_{\lambda}^{v}\left(v_{\lambda}\right)=v_{\lambda-\nu} \otimes v+$ terms of lower weight in the first factor. 
- For any $w, w^{\prime} \in \mathcal{W}, w \neq w^{\prime}$, and any $\nu \in P(V)$, the vector $w \cdot \lambda-w^{\prime} \cdot(\lambda-\nu)$ does not belong to $P(V)$.

If $\lambda=\sum_{i} \lambda_{i} \omega_{i}$, where $\omega_{i}$ are fundamental weights and $\lambda_{i}$ are large enough positive numbers then $\lambda$ is generic with respect to $V$.

Lemma 5.4 [18] Let $\lambda \in P^{+}$be generic with respect to $V$. Let $v \in V[\nu]$. Consider the intertwining operator $\Phi_{\lambda}^{v}: M_{\lambda} \rightarrow M_{\lambda-\nu} \otimes V$. For $w \in \mathcal{W}$, consider the singular vector $v_{w \cdot \lambda}^{\lambda} \in M_{\lambda}$. Then there exists a unique vector $A_{w}(\lambda)(v) \in V[w(\nu)]$ such that

$$
\Phi_{\lambda}^{v}\left(v_{w \cdot \lambda}^{\lambda}\right)=v_{w \cdot(\lambda-\nu)}^{\lambda-\nu} \otimes A_{w}(\lambda)(v)+\text { terms of lower weight in the first factor. }
$$

For generic $\lambda \in P^{+}$, Lemma 5.4 defines a linear operator $A_{w}(\lambda): V \rightarrow V$ such that $A_{w}(\lambda)(V[\nu]) \subset V[w(\nu)]$ for all $\nu \in P(V)$. This operator is extended to other values of $\lambda$ as a rational function of $\lambda$.

The collection of rational functions $A_{w}(\lambda), w \in \mathcal{W}$, is called the dynamical Weyl group acting on $V$.

Introduce new linear operators $\mathcal{A}_{w}(\lambda): V \rightarrow V$ for $w \in \mathcal{W}$. Namely, for any $w \in \mathcal{W}, \nu \in P(V), v \in V[\nu]$, set

$$
\mathcal{A}_{w}(\lambda) v=A_{w}(\lambda+\nu) v .
$$

We still have $\mathcal{A}_{w}(\lambda)(V[\nu]) \subset V[w(\nu)]$ for all $\nu \in P(V)$.

\section{Lemma $5.5[18]$}

- For any $w_{1}, w_{2} \in \mathcal{W}$ and $\nu \in P(V)$, we have

$$
\left.\left(\mathcal{A}_{w_{1}}\left(w_{2} \cdot \lambda\right) \mathcal{A}_{w_{2}}(\lambda)\right)\right|_{V[\nu]}=\left.c_{w_{1}, w_{2}, \lambda, \nu} \mathcal{A}_{w_{1} w_{2}}(\lambda)\right|_{V[\nu]},
$$

where $c_{w_{1}, w_{2}, \lambda, \nu}$ is a constant depending on $w_{1}, w_{2}, \lambda, \nu$.

- For any $w, w_{1}, w_{2} \in \mathcal{W}, \nu \in P(V)$, the limits

$$
\mathcal{A}_{w}(\infty)=\lim _{\lambda \rightarrow \infty} \mathcal{A}_{w}(\lambda), \quad c_{w_{1}, w_{2}, \nu}=\lim _{\lambda \rightarrow \infty} c_{w_{1}, w_{2}, \lambda, \nu}
$$

do exist. Therefore, we have

$$
\left.\left(\mathcal{A}_{w_{1}}(\infty) \mathcal{A}_{w_{2}}(\infty)\right)\right|_{V[\nu]}=\left.c_{w_{1}, w_{2}, \nu} \mathcal{A}_{w_{1} w_{2}}(\infty)\right|_{V[\nu]} .
$$

Moreover, the collection of operators $\mathcal{A}_{w}(\infty), w \in \mathcal{W}$, gives the canonical projective action of $\mathcal{W}$ on $V$. 
- For any vector $v \in V[\nu]$ and $w \in \mathcal{W}$, we have

$$
\mathcal{A}_{w}(\lambda) H_{i}\left(\lambda+\rho+\frac{\nu}{2}\right) v=H_{i}\left(w \cdot \lambda+\rho+\frac{w(\nu)}{2}\right) \mathcal{A}_{w}(\lambda) v .
$$

Proof The first statement follows from [18, Theorems 8 and 10]. The second statement is [18, Corollary 14]. The statement of [18, Lemma 18], which holds for any root system, gives the last statement of our lemma.

According to this lemma, if $\omega$ is an eigenvector of the operators $H_{i}\left(\lambda+\rho+\frac{\nu}{2}\right)$, then $\mathcal{A}_{w}(\lambda) \omega$ is an eigenvector of the operators $H_{i}\left(w \cdot \lambda+\rho+\frac{w(\nu)}{2}\right)$.

Let $\lambda$ be generic. Let $t$ be a solution of the Bethe Ansatz equation associated to $\left(\Lambda_{\infty}, \lambda\right)$ and let $y$ be the corresponding $r$-tuple of polynomials. Then $\omega(t)$ is an eigenvector of the operators $H_{i}\left(\lambda+\rho+\Lambda_{\infty} / 2\right)$.

By Corollary 4.12, for each element $w$ of the Weyl group, we have the descendent $w y$ of $y$ obtained via the reproduction procedure. Let $w y$ represent the tuple $t_{w}$. Moreover, if $w y$ is off-diagonal, then $t_{w}$ is a critical point associated to $\left(w \Lambda_{\infty}, w \cdot \lambda\right)$ and $\omega\left(t_{w}\right)$ is an eigenvector of the operators $H_{i}\left(w \cdot \lambda+\rho+w \Lambda_{\infty} / 2\right)$.

We conjecture that the action of the operator $\mathcal{A}_{w}(\lambda)$ coincides with the action of the Weyl group, induced by the reproduction procedure (when the latter action is well-defined). More precisely, we have

Conjecture 5.6 Let $\lambda$ be generic. Let $t$ be a critical point of the master function (2) associated to $\left(\Lambda_{\infty}, \lambda\right)$ and let $y$ be the corresponding $r$-tuple of polynomials. Let $\omega(t) \in V\left[\Lambda_{\infty}\right]$ be the corresponding Bethe vector. Let $w \in \mathcal{W}$. Assume that wy is off-diagonal. Let wy represent the tuple $t_{w}$.

Then the vector $\mathcal{A}_{w}(\lambda) \omega(t)$ is a scalar multiple of the Bethe vector $\omega\left(t_{w}\right)$.

Below we prove this conjecture for $s l_{2}$, see Theorem 5.7.

\subsection{The case of $s l_{2}$}

Let $L_{1}, \ldots, L_{n}$ be irreducible finite-dimensional $s l_{2}$ modules of highest weights $\Lambda_{1}, \ldots, \Lambda_{n} \in \mathbb{Z}_{\geq 0}$. Let $v_{1}, \ldots, v_{n}$ be the corresponding highest weight vectors. Let $V=L_{1} \otimes \cdots \otimes L_{n}$. We also fix an $n$-tuple of non-zero distinct complex numbers $z=\left(z_{1}, \ldots, z_{n}\right)$ and $l \in \mathbb{Z}_{\geq 0}$. We set $\Lambda_{\infty}=\sum_{s=1}^{n} \Lambda_{s}-2 l$. 
In the case of $s l_{2}$ the weight function $\omega(t)$ can be rewritten in the following form. We say $m=\left(m_{1}, \ldots, m_{n}\right) \in \mathcal{C}\left(\Lambda, \Lambda_{\infty}\right)$ if $m_{s} \in\left\{0, \ldots, \Lambda_{i}\right\}, s=1, \ldots, n$, and $\sum_{s=1}^{n} m_{s}=l$. Set

$$
\omega_{m}(t)=\left(\prod_{j=1}^{n}\left(m_{j} !\right)^{-1}\right) \operatorname{Sym} \prod_{s=1}^{n} \prod_{i=m_{1}+\cdots+m_{s-1}+1}^{m_{1}+\cdots+m_{s}} \frac{1}{t_{i}-z_{s}},
$$

where Sym denotes the symmetrization with respect to $t_{1}, \ldots, t_{l}$. Let

$$
F^{m} v:=F^{m_{1}} v_{1} \otimes \cdots \otimes F^{m_{n}} v_{n} .
$$

Then we explicitly have

$$
\omega(t)=\sum_{m \in \mathcal{C}\left(\Lambda, \Lambda_{\infty}\right)} \omega_{m}(t) F^{m} v
$$

Recall that if $t$ is a critical point of the master function (2) then the vector $\omega(t)$ is called the Bethe vector.

It follows from [16], that there exists a Zariski open set $U_{1}=U(\Lambda)$ in $\mathbb{C}^{n}$ such that for any $z \in U_{1}$ there exists a Zariski open set $U_{2}=U_{2}(\Lambda, z)$ in $\mathbb{C}$ such for all $\lambda \in U_{2}(z)$, the number of orbits of critical points of the $s l_{2}$ master function (2) associated to $\left(\Lambda_{\infty}, \lambda\right)$ equals to the dimension of the subspace of $V$ of vectors of weight $\Lambda_{\infty}=\sum_{s=1}^{n} \Lambda_{s}-2 l$. Moreover all critical points are non-degenerate and the corresponding Bethe vectors form a basis in this subspace.

Theorem 5.7 Let $w$ be the generator of the $s l_{2}$ Weyl group. For $z \in U_{1}$, there exists a Zariski open set $U_{3}(z) \subset \mathbb{C}$ with the following property. Let $\lambda \in U_{3}(z)$, let $t$ be a critical point associated to $\left(\Lambda_{\infty}, \lambda\right)$ and let $y$ be the corresponding polynomial. Let $t_{w}$ be the tuple represented by the polynomial wy.

Then all roots of the polynomial wy are simple and the vector $\mathcal{A}_{w}(\lambda) \omega(t)$ is a non-zero scalar multiple of the Bethe vector $\omega\left(t_{w}\right)$.

Proof In the $s l_{2}$ case $\nu, \mu \in \mathbb{C}$, and the Casimir operator is given by $C=h \otimes h / 2+$ $e \otimes f+f \otimes e$.

We claim that the joint spectrum of $H_{k}\left(\lambda+\rho+\Lambda_{\infty} / 2\right), k=1, \ldots, n$, acting in $V\left[\Lambda_{\infty}\right]$, is generically simple. Indeed, in the limit $\lambda \rightarrow \infty$ the main term is given by the operators $\lambda h^{(k)}$. The joint spectrum of commuting operators $h^{(k)}, k=1, \ldots, n$, is simple. Therefore $H_{k}\left(\lambda+\rho+\Lambda_{\infty} / 2\right), k=1, \ldots, n$, for generic $\lambda$ have a simple joint spectrum as well.

It follows that the dynamical Weyl group maps the Bethe vectors to the Bethe vectors. 
Now we compare the two actions. We do it in the same limit $\lambda \rightarrow \infty$.

The common eigenvectors of operators $h^{(i)}$ are monomial vectors $F^{m} v$. The Weyl group of $s l_{2}$ is generated by the element $w, w^{2}=i d$ which acts on the weight vectors by

$$
w\left(F^{m_{1}} v_{1} \otimes \cdots \otimes F^{m_{n}} v_{n}\right)=c F^{\Lambda_{1}-m_{1}} v_{1} \otimes \cdots \otimes F^{\Lambda_{n}-m_{n}} v_{n},
$$

where $c$ is some non-zero constant depending on $m_{i}, \Lambda_{i}$.

By Lemma 5.5, the limit $\lambda \rightarrow \infty$, the dynamical Weyl group action on the Bethe vectors coincides (up to a scalar) with the action of the Weyl group (17).

Let us consider the limit of the action defined in terms of the reproduction procedure. It is shown in the proof of Theorem (3.2) that if $y$ represents an $s l_{2}$ critical point and $\tilde{y}$ is the immediate descendent, then for almost all $\lambda, y, \tilde{y}$ can be included in a family of critical points $y_{a}$, and their descendents $\tilde{y}_{a}$ and in the limit $\lambda_{a} \rightarrow \infty$ the product $y_{a} \tilde{y}_{a}$ tends to $T=\prod_{i=1}^{n}\left(t-z_{i}\right)^{\Lambda_{i}}$.

Finally we claim that if the polynomials $y_{a}$ of degree $l=\sum_{i=1}^{n} m_{i}$, represent critical points associated to $\lambda_{a}$ and the limit of $y_{a}$ as $\lambda_{a}$ tend to $\infty$ is $\prod_{i}\left(x-z_{i}\right)^{m_{i}}$, then the corresponding Bethe vectors tend to a scalar multiple of the monomial vector $F^{m} v$.

For $i=1, \ldots, l$, let $s(i) \in\{1, \ldots, n\}$ be such that the $i$-th root of $y, t_{i}$, tends to $z_{s(i)}$. Then we write $t_{i}(\lambda)=z_{s(i)}+c_{i} / \lambda+o(1 / \lambda)$. The Bethe Ansatz equation for $t_{i}$ implies that for any $j=1, \ldots, n$, the set of $\left\{c_{i} \mid s(i)=j\right\}$ satisfy the Bethe Ansatz equation with $n=1$ :

$$
-\frac{\Lambda_{j}}{c_{i}}+\sum_{k, k \neq i, s(k)=s(i)} \frac{2}{c_{i}-c_{k}}=1 .
$$

These equations are solved explicitly. The solutions are limits of [19, formulas (1.3.2)] as $\beta \rightarrow \infty$. It follows that all $c_{i}$ with $s(i)=j$ are different from zero and from each other.

Now consider the limit of the corresponding Bethe vector. The dominant term is

$$
\lambda^{l} \prod_{j=1}^{n}\left(m_{j} !\right)^{-1} \prod_{i=1}^{l} c_{i}^{-1} F^{m} v .
$$

This finishes the proof of the claim and the theorem. 


\section{Exponential populations}

We considered in detail the trigonometric Gaudin model, where the Bethe Ansatz equation takes the form (3). There are other models, where the reproduction procedure for the solutions of the Bethe Ansatz equation works in the same way and one obtains a transitive and free Weyl group action on each population. One such model, the quasiperiodic Gaudin model, is considered in this section, another one, the quasi-periodic XXX model, is considered in Section 7.

We fix our $\mathfrak{g}, \Lambda, \Lambda_{\infty}, l, \lambda$ as in Section 2.2. Let $z_{1}, \ldots, z_{n}$ be any distinct complex numbers. Consider the master function with exponents

$$
\begin{gathered}
\Phi^{\exp }\left(t ; \Lambda_{\infty} ; \lambda\right)=\prod_{i=1}^{r} \prod_{j=1}^{l_{i}} e^{-\left(\lambda, \alpha_{i}\right) t_{j}^{(i)}} \prod_{i=1}^{r} \prod_{j=1}^{l_{i}} \prod_{s=1}^{n}\left(t_{j}^{(i)}-z_{s}\right)^{-\left(\Lambda_{s}, \alpha_{i}\right)} \times \\
\prod_{i=1}^{r} \prod_{1 \leq j<s \leq l_{i}}\left(t_{j}^{(i)}-t_{s}^{(i)}\right)^{\left(\alpha_{i}, \alpha_{i}\right)} \prod_{1 \leq i<j \leq r} \prod_{s=1}^{l_{i}} \prod_{k=1}^{l_{j}}\left(t_{s}^{(i)}-t_{k}^{(j)}\right)^{\left(\alpha_{i}, \alpha_{j}\right)} .
\end{gathered}
$$

We call $t=\left(t_{j}^{(i)}\right)_{i=1, \ldots, r}^{j=1 \ldots l_{i}}$ a critical point of the master function with exponents associated to $\left(\Lambda_{\infty}, \lambda\right)$ if

(19) $-\left(\lambda, \alpha_{i}\right)-\sum_{s=1}^{n} \frac{\left(\Lambda_{s}, \alpha_{i}\right)}{t_{j}^{(i)}-z_{s}}+\sum_{s, s \neq i} \sum_{k=1}^{l_{s}} \frac{\left(\alpha_{s}, \alpha_{i}\right)}{t_{j}^{(i)}-t_{k}^{(s)}}+\sum_{s, s \neq j} \frac{\left(\alpha_{i}, \alpha_{i}\right)}{t_{j}^{(i)}-t_{s}^{(i)}}=0$,

for $i=1, \ldots, r, j=1, \ldots, l_{i}$.

We have analogs of Propositions 5.2 and 5.3.

Proposition 6.1 Let $\mathfrak{g}=s l_{N+1}$. For almost all $\lambda$ the number of orbits of critical points of the master function with exponents associated to $\left(\Lambda_{\infty}, \lambda\right)$ and counted with multiplicities does not exceed the dimension of the subspace of the weight $\Lambda_{\infty}$ in the tensor product $L_{\Lambda_{1}} \otimes \cdots \otimes L_{\Lambda_{n}}$.

Proof The number of critical points of the master function with exponents (18) is finite for almost all $\lambda$, see [6].

Replacing the factors $e^{-\left(\lambda, \alpha_{i}\right) t_{j}^{(i)}}$ in the master function (18) with $\left(1+t_{j}^{(i)} / m\right)^{-\left(\lambda, \alpha_{i}\right) m}$ we obtain a master function of type (2). Therefore the function (18) is the limit of master functions of type (2) as $m \rightarrow \infty$. The proposition now follows from Proposition 5.2 and the fact that the number of orbits of isolated critical points of a function counted with multiplicity does not change under small deformations of the function. 
A different proof of Proposition 6.1 which uses Schubert Calculus is given in [6].

Proposition 6.2 Let $\mathfrak{g}=s l_{2}$. For almost all $\lambda \in \mathbb{C}$ and almost all $\left(z_{1}, \ldots, z_{n}\right) \in \mathbb{C}^{n}$, the number of orbits of critical points of the master function with exponents associated to $\left(\Lambda_{\infty}, \lambda\right)$ equals the dimension of the subspace of weight $\Lambda_{\infty}$ in the tensor product $L_{\Lambda_{1}} \otimes \cdots \otimes L_{\Lambda_{n}}$. Moreover all these points are non-degenerate.

Proof If $\lambda$ is a large positive integer then the proposition is proved by using methods of [12]. The rest is similar to the proof of Proposition 5.3

Let $\mathfrak{g}$ be a Kac-Moody algebra. As in Section 3.2 we represent a tuple $t=\left(t_{j}^{(i)}\right)_{i=1, \ldots, r}^{j=1, \ldots, l_{i}}$ by the $r$-tuple of polynomials $y=\left(y_{1}, \ldots, y_{r}\right)$, where $y_{i}=\prod_{j=1}^{l_{i}}\left(x-t_{j}^{(i)}\right), i=1, \ldots, r$. We make no distinction between $\left(y_{1}, \ldots, y_{r}\right)$ and $\left(c_{1} y_{1}, \ldots, c_{r} y_{r}\right)$ where $c_{1}, \ldots, c_{r}$ are non-zero complex numbers. We introduce polynomials $T_{i}, i=1, \ldots, r$, by formula (7).

We call an $r$-tuple of polynomials y exponentially off-diagonal if its roots do not belong to the union of singular hyperplanes in (19). Namely $y$ is exponentially off-diagonal if for $i=1, \ldots, r$, all roots of the polynomial $y_{i}$ are simple, different from the roots of the polynomials $y_{j}$ for all $j$ such that $\left(\alpha_{j}, \alpha_{i}\right) \neq 0$ and different from the roots of the polynomial $T_{i}$.

We have the corresponding exponential reproduction procedure. Namely, an $r$-tuple of polynomials $y$ is called exponentially fertile in the $i$-th direction with respect to $\lambda$, $i \in\{1, \ldots, r\}$, if there exists a polynomial $\tilde{y}_{i}$ such that

$$
W\left(y_{i}, e^{\left\langle\lambda, \alpha_{i}^{\vee}\right\rangle \tilde{y}_{i}}\right)=e^{\left\langle\lambda, \alpha_{i}^{\vee}\right\rangle x} T_{i} \prod_{j=1, j \neq i}^{r} y_{j}^{-a_{i j}} .
$$

Then the $r$-tuple of polynomials $y^{(i)}=\left(y_{1}, \ldots, \tilde{y}_{i}, \ldots, y_{r}\right)$ is called an exponential immediate descendent of $y$ with respect to $\lambda$ in the direction $i$.

An $r$-tuple of polynomials is called exponentially fertile with respect to $\lambda$ if it is exponentially fertile with respect to $\lambda$ in all directions $i=1, \ldots, r$.

Theorem 6.3 Let $\mathfrak{g}$ be a Kac-Moody algebra. An $r$-tuple of polynomials y represents a $\mathfrak{g}$ critical point of the master function with exponents associated to $\left(\Lambda_{\infty}, \lambda\right)$ if and only if $\operatorname{deg} y_{i}=l_{i}, y$ is exponentially off-diagonal and exponentially fertile with respect to $\lambda$. Moreover, if $y$ represents a critical point of the master function with exponents associated to $\left(\Lambda_{\infty}, \lambda\right)$ and if the immediate descendent of $y$ with respect $\lambda$ in the $i$-th direction, $y^{(i)}=\left(y_{1}, \ldots, \tilde{y}_{i}, \ldots, y_{r}\right)$, is exponentially off-diagonal then $y^{(i)}$ represents a critical point of the master function with exponents associated to $\left(s_{i} \Lambda_{\infty}, s_{i} \lambda\right)$. 
Proof The proof is similar to the proof of Theorem 3.5.

An $r$-tuple of polynomials $y^{\left(i_{1}, i_{2}, \ldots, i_{m}\right)}$, where $i_{k} \in\{1, \ldots, r\}, k=1, \ldots, m$, is called an exponential descendent of $y$ with respect to $\lambda$ in the directions $\left(i_{1}, \ldots, i_{m}\right)$ if there exist $r$-tuples of polynomials $y^{\left(i_{1}, i_{2}, \ldots, i_{k}\right)}, k=1, \ldots, m-1$, such that for $k=1, \ldots, m$, the $r$-tuple $y^{\left(i_{1}, i_{2}, \ldots, i_{k}\right)}$ is an exponential immediate descendent of $y^{\left(i_{1}, i_{2}, \ldots, i_{k-1}\right)}$ with respect to $s_{i_{k-1}} \ldots s_{i_{2}} s_{i_{1}} \lambda$ in the $i_{k}$-th direction.

An $r$-tuple of polynomials $y$ is called exponentially super-fertile with respect to $\lambda$ if it is exponentially fertile with respect to $\lambda$ and all exponential descendents $y^{\left(i_{1}, i_{2}, \ldots, i_{m}\right)}$ of $y$ with respect to $\lambda$ in the directions $\left.\left(i_{1}, \ldots, i_{m}\right)\right)$ are exponentially fertile with respect to $s_{i_{m}} \ldots s_{i_{1}} \lambda$.

For any $N$-tuple of functions $y$ and an $s l_{N+1}$ weight $\lambda$, we set $y_{N+1}=1$ and define the linear differential operator of order $N+1$ :

$$
D^{\exp }(y, \lambda)=\prod_{i}^{N \rightarrow 0}\left(\partial-\ln ^{\prime}\left(\frac{y_{i+1} \prod_{s=1}^{i} e^{\left(\lambda, \alpha_{s}\right) x} T_{s}}{y_{i}}\right)\right) .
$$

Proposition 6.4 Let $y$ be an $N$-tuple of polynomials and $l_{i}=\operatorname{deg} y_{i}, i=1, \ldots, N$. Let $\Lambda_{\infty}$ be given by (1). Let $y$ represent an $s l_{N+1}$ critical point of the master function with exponents associated to $\left(\Lambda_{\infty}, \lambda\right)$ or let $y$ be exponentially super-fertile with respect to $\lambda$. Then the kernel of the operator $D^{\exp }(y, \lambda)$ is spanned by functions of the form

$$
p_{0}, p_{1} e^{\left(\lambda, \alpha_{1}\right) x}, \ldots, p_{N} e^{\left(\lambda, \alpha_{1}+\cdots+\alpha_{N}\right) x},
$$

where $p_{i}$ is a polynomial of degree deg $y_{1}+\left(\Lambda_{\infty}, \alpha_{1}+\cdots+\alpha_{i}\right)$. The only singular points of the operator $D^{\exp }(y, \lambda)$ in $\mathbb{C}$ are regular singular points located at $z_{1}, \ldots, z_{n}$, and the exponents at $z_{i}, i=1, \ldots, n$, are

$$
z_{i}: 0,\left(\Lambda_{i}+\rho, \alpha_{1}\right),\left(\Lambda_{i}+\rho, \alpha_{1}+\alpha_{2}\right), \ldots,\left(\Lambda_{i}+\rho, \alpha_{1}+\cdots+\alpha_{N}\right),
$$

Conversely, if a linear differential operator $D$ of order $N+1$ has the kernel spanned by functions of the form (20) and the only singular points of $D$ in $\mathbb{C}$ are regular singular points at $z_{1}, \ldots, z_{n}$ with the exponents given by (21), then the $N$-tuple $y$ given by the divided Wronskians

$$
y_{i}=\frac{W\left(p_{0}, p_{1} e^{\left(\lambda, \alpha_{1}\right) x}, \ldots, p_{i-1} e^{\left(\lambda, \sum_{j=1}^{i-1} \alpha_{j}\right) x}\right)}{e^{\left(\lambda, \sum_{j=1}^{i-1}(i-j) \alpha_{j}\right) x} \prod_{j=1}^{i-1} T_{j}^{i-j}},
$$

$i=1, \ldots, N$, is an $N$-tuple of polynomials which is exponentially super-fertile with respect to $\lambda$ and satisfies $\operatorname{deg} y_{i}=l_{i}, i=1, \ldots, N$. 
Proof The proof is similar to the proof of Corollary 4.4.

Conjecture 6.5 If an $r$-tuple of polynomials y represents a critical point of the master function with exponents associated to $\left(\Lambda_{\infty}, \lambda\right)$ then $y$ is exponentially super-fertile with respect to $\lambda$.

Theorem 6.6 Conjecture 6.5 holds for the case of simple Lie algebras of types $A_{N}$ and $B_{N}$.

Proof The proof is similar to the proof of Theorems 4.5, 4.8.

For an $r$-tuple of polynomials $y$ and a $\mathfrak{g}$ weight $\lambda$, we denote $P^{\exp }(y, \lambda)$ the set of all pairs of the form $\left(y^{\left(i_{1}, i_{2}, \ldots, i_{m}\right)}, s_{i_{m}} \ldots s_{i_{2}} s_{i_{1}} \lambda\right)$, where $m \in \mathbb{Z}_{\geq 0}, i_{k} \in\{1, \ldots, r\}$, $k=1, \ldots, m$, and $y^{\left(i_{1}, i_{2}, \ldots, i_{m}\right)}$ is an exponential descendent of $y$ with respect to $\lambda$ in the directions $\left(i_{1}, \ldots, i_{m}\right)$.

We call the set $P^{\exp }(y, \lambda)$ the exponential prepopulation originated at $(y, \lambda)$. Let an $r$-tuple of polynomials $y$ be exponentially super-fertile with respect to $\lambda$. We call the set $P^{\exp }(y, \lambda)$ the exponential population originated at $(y, \lambda)$.

Theorem 6.7 Let $\mathfrak{g}$ be any simple Lie algebra and let $\lambda$ be a strongly non-integral $\mathfrak{g}$-weight. Let an $r$-tuple of polynomials $y$ be exponentially super-fertile with respect to $\lambda$. Then the map $P^{\exp }(y, \lambda) \rightarrow \mathcal{W} \lambda$ such that $(\tilde{y}, \tilde{\lambda}) \mapsto \tilde{\lambda}$ is a bijection of the exponential population originated at $(y, \lambda)$ and of the orbit of the Weyl group.

Proof The proof is similar to the proof of Corollary 4.12.

\section{Difference reproduction}

In this section we describe the Bethe Ansatz equation corresponding to the quasi-periodic XXX model. In this case the reproduction procedure works in a similar way and one obtains a free and transitive Weyl group action on a population.

Let $h \in \mathbb{C}$ be a complex non-zero number. We fix $\mathfrak{g}, \Lambda, \Lambda_{\infty}, l, \lambda$ as in Section 2.2. Let $z_{1}, \ldots, z_{n}$ be any distinct complex numbers, subject to the conditions $z_{i}-z_{j} \notin h \mathbb{Z}$ for all $i, j \in\{1, \ldots, n\}, i \neq j$. 
Consider the exponential XXX Bethe equation on variables $t=\left(t_{j}^{(i)}\right)_{i=1, \ldots, r}^{j=1, \ldots, l_{i}}$ :

$$
\begin{aligned}
e^{\left\langle\lambda, \alpha_{i}^{\vee}\right\rangle h}= & \prod_{s=1}^{n} \frac{t_{j}^{(i)}-z_{s}+\left(\Lambda_{s}, \alpha_{i}\right) h / 2}{t_{j}^{(i)}-z_{s}-\left(\Lambda_{s}, \alpha_{i}\right) h / 2} \times \\
& \prod_{\substack{m=1, \ldots, r, m \neq i}}\left(\prod_{k=1}^{l_{m}} \frac{t_{j}^{(i)}-t_{k}^{(m)}+h / 2}{t_{j}^{(i)}-t_{k}^{(m)}-h / 2}\right)^{-a_{i m}} \prod_{\substack{k=1, \ldots, l i \\
k \neq j}} \frac{t_{j}^{(i)}-t_{k}^{(i)}-h}{t_{j}^{(i)}-t_{k}^{(i)}+h},
\end{aligned}
$$

where $i=1, \ldots, r, j=1, \ldots, l_{i}$.

As in Section 3.2 we represent a tuple $t=\left(t_{j}^{(i)}\right)_{i=1, \ldots, r}^{j=1, \ldots, l_{i}}$ by the $r$-tuple of polynomials $y=\left(y_{1}, \ldots, y_{r}\right)$, where $y_{i}=\prod_{j=1}^{l_{i}}\left(x-t_{j}^{(i)}\right), i=1, \ldots, r$. We make no distinction between $\left(y_{1}, \ldots, y_{r}\right)$ and $\left(c_{1} y_{1}, \ldots, c_{r} y_{r}\right)$ where $c_{1}, \ldots, c_{r}$ are non-zero complex numbers.

For $i=1, \ldots, r$, set

$$
T_{i}^{(h)}(x)=\prod_{s=1}^{n} \prod_{j=1}^{\left(\Lambda_{s}, \alpha_{i}\right)}\left(x-z_{s}-\left(\Lambda_{s}, \alpha_{i}\right) h / 2+j h\right) .
$$

An $r$-tuple of polynomials $y$ is called exponentially difference off-diagonal with respect to $(\Lambda, z ; h)$ if for $i=1, \ldots, r$ the polynomial $y_{i}(x)$ has only simple roots, different from the roots of polynomials $y_{m}(x+h / 2)$, whenever $\left(\alpha_{i}, \alpha_{m}\right) \neq 0$, and different from the roots of polynomials $T_{i}^{(h)}, y_{i}(x+h)$.

A solution $t$ of (22) is called off-diagonal if the corresponding $r$-tuple of polynomials is exponentially difference off-diagonal.

Lemma 7.1 A polynomial $y$ of degree $l$ represents an $s l_{2}$ off-diagonal solution of exponential XXX Bethe equation associated to $\left(\Lambda_{\infty}, \lambda\right)$ if and only if $y$ is exponentially difference off-diagonal and there exists a polynomial $B(x)$ such that

$$
\begin{aligned}
y(x+h) e^{\left\langle\lambda, \alpha^{\vee}\right\rangle h} & \prod_{s=1}^{n}\left(x-z_{s}-\frac{\left(\Lambda_{s}, \alpha\right) h}{2}\right) \\
& +B(x) y(x)+y(x-h) \prod_{s=1}^{n}\left(x-z_{s}+\frac{\left(\Lambda_{s}, \alpha\right) h}{2}\right)=0 .
\end{aligned}
$$

Proof The lemma is proved similarly to Lemma 3.1.

Proposition 7.2 Let $\mathfrak{g}=s l_{N+1}$. For almost all $\lambda$ the number of orbits of off-diagonal solutions of the exponential XXX Bethe Ansatz equations associated to $\left(\Lambda_{\infty}, \lambda\right)$ does not exceed the dimension of the subspace of the weight $\Lambda_{\infty}$ in the tensor product $L_{\Lambda_{1}} \otimes \cdots \otimes L_{\Lambda_{n}}$. 
Proof The proof is similar to the proof of the Proposition 6.1 with the help of $[8$, Corollary 4.15].

Proposition 7.3 [18] Let $\mathfrak{g}=s l_{2}$. For almost all $\lambda$ and almost all $\left(z_{1}, \ldots, z_{n}\right) \in \mathbb{C}^{n}$, the number of orbits of solutions $t$ of the exponential XXX Bethe Ansatz equation associated to $\left(\Lambda_{\infty}, \lambda\right)$ such that $t_{i} \neq t_{j}$ equals the dimension of the subspace of weight $\Lambda_{\infty}$ in the tensor product $L_{\Lambda_{1}} \otimes \cdots \otimes L_{\Lambda_{n}}$. Moreover all such solutions are non-degenerate.

We now describe the corresponding exponential difference reproduction procedure.

Denote $W_{h}$ the discrete Wronskian:

$$
W_{h}\left(f_{1}, \ldots, f_{N}\right):=\operatorname{det}\left(f_{i}(x+(j-1) h)\right)_{i, j=1, \ldots, N} .
$$

An $r$-tuple of polynomials $y$ is called exponentially difference fertile with respect to $\lambda$ in the $i$-th direction, $i \in\{1, \ldots, r\}$, if there exists a polynomial $\tilde{y}_{i}$ such that

$$
W_{h}\left(y_{i}, e^{\left\langle\lambda, \alpha_{i}^{\vee}\right\rangle x} \tilde{y}_{i}\right)=e^{\left\langle\lambda, \alpha_{i}^{\vee}\right\rangle x} T_{i}^{(h)}(x) \prod_{m=1, m \neq i}^{r}\left(y_{m}(x+h / 2)\right)^{-a_{i m}} .
$$

Then the $r$-tuple of polynomials $y^{(i)}=\left(y_{1}, \ldots, \tilde{y}_{i}, \ldots, y_{r}\right)$ is called an exponential difference immediate descendent of $y$ with respect to $\lambda$ in the $i$-th direction.

An $r$-tuple is called exponentially difference fertile with respect to $\lambda$ if it is exponentially difference fertile with respect to $\lambda$ in all directions $i=1, \ldots, r$.

Theorem 7.4 An $r$-tuple of polynomials y represents an off-diagonal solution of the exponential XXX Bethe Ansatz equation associated to $\left(\Lambda_{\infty}, \lambda\right)$ if and only if $y$ is exponentially difference off-diagonal, $\operatorname{deg} y_{i}=l_{i}, i=1, \ldots, r$, and $y$ is exponentially difference fertile with respect to $\lambda$. Moreover, if $y$ represents an off-diagonal solution of the exponential XXX Bethe Ansatz equation associated to $\left(\Lambda_{\infty}, \lambda\right)$ and if the exponential difference immediate descendent of $y$ with respect $\lambda$ in the $i$-th direction, $y^{(i)}=\left(y_{1}, \ldots, \tilde{y}_{i}, \ldots, y_{r}\right)$, is exponentially difference off-diagonal then $y^{(i)}$ represents an off-diagonal solution of the exponential XXX Bethe Ansatz equation associated to $\left(s_{i} \Lambda_{\infty}, s_{i} \lambda\right)$.

Proof The proof is similar to the proof of Theorem 3.5, cf [10] and also [13, 8].

An $r$-tuple of polynomials $y^{\left(i_{1}, i_{2}, \ldots, i_{m}\right)}$, where $m \in \mathbb{Z}_{\geq 0}, i_{k} \in\{1, \ldots, r\}, k=1, \ldots, m$, is called an exponential difference descendent of $y$ with respect to $\lambda$ in the directions 
$\left(i_{1}, \ldots, i_{m}\right)$ if there exist $r$-tuples of polynomials $y^{\left(i_{1}, i_{2}, \ldots, i_{k}\right)}, k=1, \ldots, m-1$, such that for $k=1, \ldots, m$, the $r$-tuple $y^{\left(i_{1}, i_{2}, \ldots, i_{k}\right)}$ is an exponential difference immediate descendent of $y^{\left(i_{1}, i_{2}, \ldots, i_{k-1}\right)}$ with respect to $s_{i_{k-1}} \ldots s_{i_{2}} s_{i_{1}} \lambda$ in the $i_{k}$-th direction.

An $r$-tuple of polynomials $y$ is called exponentially difference super-fertile with respect to $\lambda$ if it is exponentially difference fertile with respect to $\lambda$ and all exponential descendents $y^{\left(i_{1}, i_{2}, \ldots, i_{m}\right)}$ of $y$ with respect to $\lambda$ in the directions $\left(i_{1}, i_{2}, \ldots, i_{m}\right)$ are exponentially difference fertile with respect to $s_{i_{m}} \ldots s_{i_{1}} \lambda$.

For any $N$-tuple of functions $y$ and an $s l_{N+1}$ weight $\lambda$, we set $y_{N+1}=1$ and define the linear difference operator:

$$
\begin{aligned}
D_{h}^{e x p}(y, \lambda)= & \prod_{i}^{N \rightarrow 0}\left(\partial_{h}-\frac{y_{i+1}(x+(i+2) h / 2)}{y_{i+1}(x+i h / 2)} \frac{y_{i}(x+(i-1) h / 2)}{y_{i}(x+(i+1) h / 2)} \times\right. \\
& \left.\prod_{s=1}^{i} \frac{e^{h\left(\lambda, \alpha_{s}\right)} T_{s}(x+(2 i-s+1) h / 2)}{T_{s}(x+(2 i-s-1) h / 2)}\right),
\end{aligned}
$$

where $\partial_{h}$ is the operator acting on functions of $x$ by the formula $\partial_{h}(f(x))=f(x+h)$.

Let $V$ be a space spanned by functions of the type $p_{0} e^{\lambda_{0} x}, p_{1} e^{\lambda_{1} x}, \ldots, p_{N} e^{\lambda_{N} x}$ where $p_{i}$, $i=0, \ldots, N$, are polynomials and $\lambda_{i} \in \mathbb{C}, i=0, \ldots, N$. We say the space $V$ has no base points if for any $z \in \mathbb{C}$ there exists $f \in V$, such that $f(z) \neq 0$.

Assume $V$ has no base points. For $i=2, \ldots, N$, let $U_{i}$ be the monic polynomial of the greatest possible degree such that $W_{h}\left(f_{1}, \ldots, f_{i}\right) / U_{i}$ is a holomorphic function for all $f_{1}, \ldots, f_{i} \in V$. Following [8], we call an $N$-tuple of monic polynomials $\left(T_{1}, \ldots, T_{N}\right) a$ frame of space $V$ if for $i=2, \ldots, N$ we have $U_{i}=\prod_{j=1}^{i-1} \prod_{s=1}^{i-j} T_{j}(x+(s-1) h)$.

Lemma 7.5 Let $V$ be a space spanned by functions of the type

$$
p_{0} e^{\lambda_{0} x}, p_{1} e^{\lambda_{1} x}, \ldots, p_{N} e^{\lambda_{N} x} .
$$

Let $V$ have no base points. Then there exists a unique frame of $V$.

Proof The proof is similar to the proof of [8, Lemma 4.9].

Proposition 7.6 Let $y$ be an $N$-tuple of polynomials and $l_{i}=\operatorname{deg} y_{i}, i=1, \ldots, N$. Let $\Lambda_{\infty}$ be given by (1). Let y represent an off-diagonal solution of $s l_{N+1}$ exponential XXX Bethe Ansatz equation associated to $\left(\Lambda_{\infty}, \lambda\right)$ or let $y$ be exponentially difference super-fertile with respect to $\lambda$. Then the kernel of the operator $D_{h}^{\exp }(y, \lambda)$ is spanned by functions of the form

$$
p_{0}, p_{1} e^{\left(\lambda, \alpha_{1}\right) x}, \ldots, p_{N} e^{\left(\lambda, \alpha_{1}+\cdots+\alpha_{N}\right) x},
$$


where $p_{i}$ is a polynomial of degree $\operatorname{deg} y_{1}+\left(\Lambda_{\infty}, \alpha_{1}+\cdots+\alpha_{i}\right)$. Moreover, the $N$-tuple

$$
\left(T_{1}^{(h)}(x), T_{2}^{(h)}(x+h / 2), \ldots, T_{N}^{(h)}(x+(N-1) h / 2)\right)
$$

is the frame of the kernel of the operator $D_{h}^{\exp }(y, \lambda)$.

Conversely, if a linear difference operator $D$ of order $N+1$ has the kernel spanned by functions of the form (23) with the frame (24) then the $N$-tuple $y$ given by

$$
y_{i}=\frac{W_{h}\left(p_{0}, p_{1} e^{\left(\lambda, \alpha_{1}\right) x}, \ldots, p_{i-1} e^{\left(\lambda, \sum_{j=1}^{i-1} \alpha_{j}\right) x}\right)}{e^{\left(\lambda, \sum_{j=1}^{i-1}(i-j) \alpha_{j}\right) x} \prod_{s=1}^{i-j} T_{j}^{(h)}(x+(s+j / 2-3 / 2) h)},
$$

$i=1, \ldots, N$, is an $N$-tuple of polynomials which is exponentially difference superfertile with respect to $\lambda$ and satisfies $\operatorname{deg} y_{i}=l_{i}, i=1, \ldots, N$.

Proof The proof is similar to the proof of Corollary 4.4.

Conjecture 7.7 Let $\mathfrak{g}$ be any simple Lie algebra. If an $r$-tuple of polynomials $y$ represents an off-diagonal solution of the exponential XXX Bethe Ansatz equation associated to $\left(\Lambda_{\infty}, \lambda\right)$ then $y$ is exponentially difference super-fertile with respect to $\lambda$.

Theorem 7.8 Conjecture 7.7 holds for the case of simple Lie algebras of types $A_{N}$ and $B_{N}$.

Proof The proof is similar to the proof of Theorems 4.5, 4.8.

For an $r$-tuple of polynomials $y$ we denote $P_{h}^{e x p}(y, \lambda)$ the set of all pairs of the form $\left(y^{\left(i_{1}, i_{2}, \ldots, i_{m}\right)}, s_{i_{m}} \ldots s_{i_{2}} s_{i_{1}} \lambda\right)$, where $m \in \mathbb{Z}_{\geq 0}, i_{k} \in\{1, \ldots, r\}, k=1, \ldots, m$, and $y^{\left(i_{1}, i_{2}, \ldots, i_{m}\right)}$ is an exponential difference descendent of $y$ with respect to $\lambda$ in directions $\left(i_{1}, \ldots, i_{m}\right)$.

We call the set $P_{h}^{\exp }(y, \lambda)$ the exponential difference prepopulation originated at $(y, \lambda)$. If an $r$-tuple of polynomials $y$ is exponentially difference super-fertile with respect to $\lambda$, then we call the set $P_{h}^{\exp }(y, \lambda)$ the exponential difference population originated at $(y, \lambda)$.

Theorem 7.9 Let $\mathfrak{g}$ be any simple Lie algebra and let $\lambda$ be a strongly non-integral $\mathfrak{g}$-weight. Let an $r$-tuple of polynomials $y$ be exponentially difference super-fertile with respect to $\lambda$. Then the map $P_{h}^{\exp }(y, \lambda) \rightarrow \mathcal{W} \lambda$ such that $(\tilde{y}, \tilde{\lambda}) \mapsto \tilde{\lambda}$ is a bijection of the exponential difference population originated at $(y, \lambda)$ and of the orbit of the Weyl group.

Proof The proof is similar to the proof of Corollary 4.12. 


\section{References}

[1] B Belcale, E Mukhin, Multiplicity of critical points of master functions and Schubert calculus arXiv:math.QA/0503132

[2] G Felder, A Varchenko, Three formulae for eigenfunctions of integrable Schrödinger operators, Compositio Math. 107 (1997) 143-175 MR1458747

[3] E Frenkel, Opers on the projective line, flag manifolds and Bethe ansatz, Mosc. Math. J. 4 (2004) 655-705, 783 MR2119144

[4] V G Kac, Infinite-dimensional Lie algebras, third edition, Cambridge University Press, Cambridge (1990) MR1104219

[5] Y Markov, A Varchenko, Hypergeometric solutions of trigonometric KZ equations satisfy dynamical difference equations, Adv. Math. 166 (2002) 100-147 MR1882860

[6] E Mukhin, V Tarasov, A Varchenko, Bispectral and $\left(g l_{N}, g l_{M}\right)$ dualities arXiv: math.QA/0510364

[7] E Mukhin, V Tarasov, A Varchenko, Bethe eigenvectors of higher transfer matrices, J. Stat. Mech. Theory Exp. (2006) P08002, 44 pp. MR2249767

[8] E Mukhin, A Varchenko, Solutions to the XXX type Bethe ansatz equations and flag varieties, Cent. Eur. J. Math. 1 (2003) 238-271 MR1993451

[9] E Mukhin, A Varchenko, Critical points of master functions and flag varieties, Commun. Contemp. Math. 6 (2004) 111-163 MR2048778

[10] E Mukhin, A Varchenko, Discrete Miura Opers and Solutions of the Bethe Ansatz Equations arXiv:math.QA/0401137

[11] E Mukhin, A Varchenko, Miura opers and critical points of master functions, Cent. Eur. J. Math. 3 (2005) 155-182 MR2129922

[12] E Mukhin, A Varchenko, Norm of a Bethe vector and the Hessian of the master function, Compos. Math. 141 (2005) 1012-1028 MR2148192

[13] E Mukhin, A Varchenko, Populations of solutions of the XXX Bethe equations associated to Kac-Moody algebras, from: "Infinite-dimensional aspects of representation theory and applications", Contemp. Math. 392, Amer. Math. Soc., Providence, RI (2005) 95-102 MR2189873

[14] N Reshetikhin, A Varchenko, Quasiclassical asymptotics of solutions to the $K Z$ equations, from: "Geometry, topology, \& physics", Conf. Proc. Lecture Notes Geom. Topology, IV, Int. Press, Cambridge, MA (1995) 293-322 MR1358621

[15] V V Schechtman, A N Varchenko, Arrangements of hyperplanes and Lie algebra homology, Invent. Math. 106 (1991) 139-194 MR1123378

[16] I Scherbak, Critical points of functions, sl 2 representations and Fuchsian differential equations with only univalued solutions arXiv:math.QA/0112269 
[17] G Szegó, Orthogonal polynomials, third edition, Amer. Math. Soc. Colloquium Publications 23, Amer. Math. Soc. (1967) MR0310533

[18] V Tarasov, A Varchenko, Difference equations compatible with trigonometric KZ differential equations, Internat. Math. Res. Notices (2000) 801-829 MR1780748

[19] A Varchenko, Critical points of the product of powers of linear functions and families of bases of singular vectors, Compositio Math. 97 (1995) 385-401 MR1353281

Department of Mathematical Sciences, Indiana University - Purdue University Indianapolis 402 North Blackford St, Indianapolis, IN 46202-3216, USA

Department of Mathematics, University of North Carolina at Chapel Hill

Chapel Hill, NC 27599-3250, USA

mukhin@math.iupui.edu, anv@email.unc.edu

Received: 18 February 2006 Revised: 4 September 2006 